\title{
ARTICLE OPEN \\ Oxidized mitochondrial DNA sensing by STING signaling promotes the antitumor effect of an irradiated immunogenic cancer cell vaccine
}

\author{
Chunju Fang ${ }^{1,2}$, Fei Mo ${ }^{1}$, Li Liu ${ }^{1}$, Jing $\mathrm{Du}^{1}$, Min Luo ${ }^{1}{ }^{1}$, Ke Men ${ }^{1}$, Feifei Na ${ }^{1}$, Wei Wang ${ }^{1}$, Hanshuo Yang ${ }^{1}$ and Xiawei Wei ${ }^{1}$
}

\begin{abstract}
Exposure to ionizing radiation, a physical treatment that inactivates live tumor cells, has been extensively applied to enhance the antitumor responses induced by cancer cell vaccines in both animal research and human clinical trials. However, the mechanisms by which irradiated cells function as immunogenic tumor vaccines and induce effective antitumor responses have not been fully explored. Here, we demonstrate that oxidized mitochondrial DNA (mtDNA) and stimulator of interferon genes (STING) signaling play a key roles in the enhanced antitumor effect achieved with an irradiated tumor cell vaccine. Elevations in ROS and oxidized mtDNA 8-OHG content could be induced in irradiated tumor cells. Oxidized mtDNA derived from irradiated tumor cells gained access to the cytosol of dendritic cells (DCs). Oxidized mtDNA, as a DAMP or adjuvant, activated the STING-TBK1-IRF3-IFN- $\beta$ pathway in DCs, which subsequently cross-presented irradiated tumor cell-derived antigens to CD8 ${ }^{+} \mathrm{T}$ cells and elicited antitumor immunity. The results of our study provide insight into the mechanism by which an irradiated cell vaccine mediates antitumor immunity, which may have implications for new strategies to improve the efficacy of irradiated vaccines.
\end{abstract}

Keywords: Irradiated tumor cell vaccine; Oxidized mitochondrial DNA; STING signaling

Cellular \& Molecular Immunology (2021) 18:2211-2223; https://doi.org/10.1038/s41423-020-0456-1

\section{INTRODUCTION}

As a form of immunotherapy, cancer vaccines have demonstrated objective therapeutic benefits in several randomized clinical trials. ${ }^{1,2}$ The activation of tumor-specific immune effects by cancer vaccines, particularly whole-tumor cell vaccines that contain all potential tumor antigens, represents a promising approach for cancer therapy. ${ }^{3}$ lonizing radiation (IR), which inactivates live tumor cells to create cancer cell vaccines, has been used extensively to enhance antitumor immunity in both animal models and human clinical trials. ${ }^{4-8}$ In addition, local radiotherapy used alone or in combination with surgery, chemotherapy, or targeted therapy is employed to treat the primary and metastatic tumors of cancer patients. ${ }^{9-11}$ Furthermore, localized radiotherapy has also been shown to induce abscopal effects in several types of cancer. ${ }^{12,13}$ Overall, ionizing radiation can potentiate the antitumor response induced by either an irradiated tumor cell vaccine or local radiotherapy.

The potential mechanisms by which ionizing radiation enhance the antitumor response induced by either an irradiated tumor cell vaccine or local radiotherapy have been explored but remain largely unknown., 14-18 It has been demonstrated that cell-surface exposure to calreticulin (CRT) is important for establishing the immunogenicity of tumor cell death elicited by irradiation, which is involved in the enhanced antitumor effect of irradiated tumor cells. ${ }^{7,16}$ Regarding local radiotherapy of tumors, some studies have described how local irradiation affects the tumor microenvironment. One report found that regulation of the Fas/Fas ligand pathway in tumor cells by irradiation plays an important role in their sensitization to antigen-specific CTLs (cytotoxic T lymphocytes) ${ }^{17}$. Another report noted that directed radiotherapy increases MHC class I expression on tumor cells, leading to increased sensitivity to antigen-specific CTLs and an immunemediated abscopal effect. ${ }^{14}$ Recently, the adaptor protein STING (stimulator of interferon genes) has garnered considerable interest given its roles in sensing DNA through cGAS (cyclic GMP-AMP synthase) in DCs in response to locally irradiated tumor cells and activating the STING pathway. ${ }^{15,18}$ However, it remains unknown why irradiation can trigger the activation of the STING pathway. Additionally, the previous work did not address the potential role of mitochondrial DNA (mtDNA) or oxidized mtDNA in the activation of the STING pathway in response to locally irradiated tumor cells.

Recently, the concept of cell damage-associated molecular patterns (DAMPs), which are endogenous molecules released by cells undergoing abnormal cell death (e.g., during pathological insult) that are capable of activating the innate immune response, ${ }^{19}$ has been highlighted. Among DAMPs, mtDNA, which is similar to its bacterial ancestor, is considered an immunostimulatory agent; mtDNA consists of a circular loop and contains significant levels of unmethylated DNA in the form of CpG

\footnotetext{
'Laboratory of Aging Research and Cancer Drug Target, State Key Laboratory of Biotherapy, National Clinical Research Center for Geriatrics, West China Hospital, Sichuan University, 610041 Chengdu, P.R. China and ²Department of Oncology, Guizhou Provincial People's Hospital, 550002 Guiyang, Guizhou, P.R. China

Correspondence: Xiawei Wei (xiaweiwei@scu.edu.cn)

These authors contributed equally: Chunju Fang, Fei Mo, Li Liu
}

Received: 30 November 2019 Accepted: 21 April 2020

Published online: 12 May 2020 
islands. $^{20}$ Given that mtDNA lacks the repair proteins and protective histones found in nuclear DNA (nDNA), mtDNA is more easily attacked by reactive oxygen species (ROS), ${ }^{21,22}$ which are byproducts of mitochondrial respiration. ${ }^{23}$ The oxidized base 8hydroxyguanosine (8-OHG), a marker of oxidative DNA damage, potentiates cytosolic immune recognition by decreasing mtDNA susceptibility to $3^{\prime}$ repair exonuclease 1 (TREX1)-mediated degradation. ${ }^{24}$ Enhanced immune sensing, which is induced by oxidized genomic DNA damaged by UV rays, is dependent on STING signaling. ${ }^{24}$ Another study ${ }^{25}$ demonstrated that herpesvirus infection induced mtDNA stress, which led to the activation of antiviral innate immune responses through the CGAS-STING pathway. In addition, oxidized mitochondrial nucleoids released by neutrophils drive type I interferon production in lupus. ${ }^{26,27}$ However, the role of mtDNA or oxidized mtDNA in the activation of antitumor immunity remains unexplored. Exposure of cells to ionizing radiation can cause the formation of radicals and ROS that can oxidize and damage DNA. ${ }^{28}$ Based on the findings mentioned above, we hypothesize that the enhanced immunogenicity of irradiated cancer cell vaccines may result from the induction of oxidized mtDNA, which in turn triggers the activation of the STING pathway.

In the present study, we demonstrate that elevations in ROS and oxidized mtDNA 8-OHG content can be induced in irradiated tumor cells, which are subsequently engulfed by DCs. We highlight the role of the oxidized mtDNA-STING pathway in the enhanced antitumor effect achieved with an irradiated cell vaccine. Our study provides insight to better understand the mechanism underlying tumor regression mediated by the irradiated cell vaccine and may have implications for new strategies to potentiate irradiated vaccine efficacy in cancer patients.

\section{MATERIALS AND METHODS}

Cell lines

A mouse colon cancer cell line (CT26), mouse Lewis lung carcinoma line (LL2) and OVA-expressing mouse thymoma cell line (EG7) were purchased from the American Type Culture Collection and maintained at $37^{\circ} \mathrm{C}$ in $5 \% \mathrm{CO}_{2}$. CT26 cells were cultured in RPMI 1640 medium (Gibco) with $10 \%$ fetal bovine serum (FBS, Invitrogen). LL2 cells were cultured in Dulbecco's modified Eagle's medium with 10\% FBS. EG7 cells were maintained in complete RPMI 1640 medium supplemented with G418 (0.4 mg/ml; InvivoGen).

\section{Mice}

Six-week to eight-week-old $\mathrm{C} 57 \mathrm{BL} / 6$ or $\mathrm{BALB} / \mathrm{c}$ mice purchased from Vital River (Peking, China) were maintained under specific pathogen-free conditions. Tmem $173^{-/-}$mice and OVA-specific T cell receptor-transgenic OT-I mice were obtained from The Jackson Laboratory. Tlr9 ${ }^{-/-}$mice were kindly provided by Bioindustry Division Oriental Yeast Co., Ltd. (Tokyo, Japan), and $I L-1 \beta^{-1-}$ mice were kindly provided by Dr. Yoichiro Iwakura of the University of Tokyo. All animal experiments were approved by the Institutional Animal Care and Use Committee of Sichuan University (Chengdu, Sichuan, China) and by the relevant animal association.

\section{Immunizations}

To inactivate live tumor cells, cells were irradiated with X-rays (Rad Source). CT26 and LL2 cells were irradiated with $100 \mathrm{~Gy}$ unless otherwise indicated. EG7 cells and mtDNA derived from untreated EG7 cells were irradiated with $75 \mathrm{~Gy}$ unless otherwise indicated. Mice were immunized subcutaneously on the left side with $1 \times 10^{6}$ irradiated tumor cells or irradiated tumor cells with mtDNA derived from untreated EG7 cells ( $5 \mu \mathrm{g}$ per mouse) as a vaccine or PBS as a control on days 0,14 , and 21 . EG7 cells were also irradiated in the presence of the ROS scavenger BHA $(100 \mu \mathrm{M}$; Sigma $)^{29,30}$ as a vaccine, and BHA was removed by washing after irradiation to rule out other nonspecific effects of BHA. In addition, we injected mice subcutaneously with 2000 IU of DNase I (Sigma) in $100 \mu \mathrm{l}$ of PBS both 3 and $18 \mathrm{~h}$ after immunization. ${ }^{31}$ One week after the three immunizations, untreated live tumor cells (CT26 or LL2 cells, $1 \times 10^{6}$; EG7 cells, $3 \times 10^{6}$ ) were injected subcutaneously into the right flank of the mice, and the tumors were measured as previously described ${ }^{2}$ according to the following formula: tumor volume $\left(\mathrm{mm}^{3}\right)=0.52 \times$ length $\times$ width $^{2}$.

Detection of cell death

To detect cell death, EG7 cells were observed under an inverted microscope (Eclipse 80i, Nikon) and were also labeled with the FITC Annexin-V Apoptosis Detection Kit with PI (\#640914; BioLegend) according to the manufacturer's instructions. EG7 cells treated with $75 \mathrm{~Gy}$ X-ray irradiation were cultured in vitro for $24 \mathrm{~h}$. CT26 cells treated with 50 Gy X-ray irradiation were cultured in vitro for $48 \mathrm{~h}$ and evaluated with the FITC Annexin-V Apoptosis Detection Kit with PI by flow cytometry. For in vivo experiments, irradiated EG7 cells in the peritoneal lavage fluid were detected after $24 \mathrm{~h}$. To analyze cell apoptosis, EG7 cells stained with rabbit anti-caspase-3 (1:400; \#9661; Cell Signaling Technology) and FITCgoat anti-rabbit lgG (1:1000; Abcam) antibodies were evaluated on a FACSAria III flow cytometer (BD Biosciences) or visualized using a Leica TCS SP5 confocal microscope after staining with DAPI.

Preparation of DNA and quantitative real-time PCR for mtDNA Mice were injected intraperitoneally with $1 \times 10^{7}$ untreated EG7 cells or irradiated EG7 cells, and PBS was used as a control. In addition, we injected mice intraperitoneally with 2000 IU of DNase I at both 3 and $18 \mathrm{~h} .{ }^{31}$ Mitochondrial DNA in the peritoneal lavage fluid and cell culture supernatants collected $24 \mathrm{~h}$ after irradiation was concentrated and purified using the QIAamp DNA Blood Mini Kit (\#51106; QIAGEN) according to the manufacturer's instructions. The quantitation of mtDNA was performed with TaqMan probes, as previously described. ${ }^{32}$ Mitochondrial DNA isolation was performed using the mtDNA Isolation Kit (\#ab65321; Abcam), and gDNA isolation was performed using the QIAamp DNA Blood Mini Kit (\#51106; QIAGEN) according to the manufacturer's instructions. To eliminate cellular proteins, mtDNA isolation was followed by phenol-chloroform extraction and isopropanol precipitation. The concentration of DNA was determined using a NanoDrop 2000 spectrophotometer (Thermo Scientific).

Mitochondrial DNA depletion

Cells were treated with dideoxycytidine (ddC; $150 \mu \mathrm{M}$; \#D5782; Sigma) for 6 days, and gDNA was extracted as described above. To measure the efficiency of mtDNA depletion, the copy number of mtDNA was normalized to that of nuclear DNA as the ratio of mtDNA encoding cytochrome $c$ oxidase $I$ to nuclear DNA encoding $18 \mathrm{~S}$ ribosomal RNA by quantitative real-time PCR. ${ }^{33,34}$

\section{Generation of BMDCs in vitro}

BMDCs were prepared as previously described. ${ }^{15,35}$ In brief, singlecell suspensions of bone marrow cells $\left(1 \times 10^{6}\right.$ cells per $\left.\mathrm{ml}\right)$ derived from C57BL/6 mice were cultured in RPMI-1640 medium containing 10\% FBS, $1 \mathrm{mM}$ sodium pyruvate, $20 \mathrm{mM}$ HEPES, $2 \mathrm{mM}$ L-glutamine, $100 \mathrm{U} / \mathrm{ml}$ penicillin $\mathrm{G}, 100 \mathrm{\mu g} / \mathrm{ml}$ streptomycin and 50 $\mu \mathrm{M} \beta$-mercaptoethanol supplemented with mouse granulocytemacrophage colony-stimulating factor (GM-CSF; $20 \mathrm{ng} / \mathrm{ml}$; PeproTech). On days 3 and 5 , half of the medium was replaced with fresh medium. Nonadherent BMDCs were harvested for a stimulation assay on day 7 in the presence of fresh GM-CSF. The percentage of $\mathrm{CD} 11 \mathrm{c}^{+}$cells in the BMDCs was evaluated by flow cytometry and found to be greater than $70 \%$.

\section{Detection of cellular ROS and 8-OHG content}

Intracellular ROS concentrations were determined using the ROS Assay Kit (\#S0033; Beyotime). After staining, EG7 cells were immediately irradiated with X-rays, incubated for $30 \mathrm{~min}$ at $37^{\circ} \mathrm{C}$ 
and analyzed by flow cytometry. To test 8-OHG oxidization induced by irradiation, EG7 cells irradiated with $75 \mathrm{~Gy}$ X-ray irradiation were cultured in vitro for $18 \mathrm{~h}$ (apoptotic EG7 cells, apoEG7 cells). The 8-OHdG levels in mtDNA and gDNA were quantified with the 8-OH-dG EIA Kit (\#ab101245; Abcam) in accordance with the manufacturer's instructions. EG7 cells were also stained with an anti-8-OHG antibody and evaluated by flow cytometry. Furthermore, to measure 8-OHG and mitochondrion content in EG7 cells, cells were stained with MitoTracker Mitochondrion-Selective according to the manufacturer's instructions (MitoTracker ${ }^{\oplus}$ Red CMXRos; \#M7512; Invitrogen), a goat anti8-OHG antibody (1:300; \#ab10802; Abcam), a FITC-donkey antigoat IgG antibody (1:1000; Thermo Fisher), and DAPI and then observed under a Leica TCS SP5 confocal microscope. Quantification of colocalization of $8-\mathrm{OHG}$ and MitoTracker based on Pearson's correlation coefficient (a perfect linear correlation is +1 ) was determined with ImageJ software.

Detection of DCs in the skin

EG7 cells were injected subcutaneously. Eighteen hours later, to detect DCs in the skin, skin tissues were excised, cut into small pieces and digested with DNase I $(0.1 \mathrm{mg} / \mathrm{ml}$; Sigma) and collagenase Type IV ( $4 \mathrm{mg} / \mathrm{ml}$; Gibco) for $2 \mathrm{~h}$ at $37^{\circ} \mathrm{C}$. The resulting cell suspensions were centrifuged, washed in PBS and passed through a 70- $\mu \mathrm{m}$ nylon mesh filter (BD Biosciences) to obtain single-cell suspensions. The single-cell suspensions were stained with PerCP-Cy ${ }^{T_{1}}$ 5.5-conjugated anti-CD45, PE-conjugated antiMHC-II, APC-conjugated anti-CD11C (1:100; BD Biosciences) and FITC-conjugated anti-IFN- $\beta$ (1:100; PBL Assay Science) antibodies for a flow cytometry assay.

Assays for apoptotic cell uptake

Briefly, 5-(and 6-)carboxyfluorescein diacetate succinimidyl ester (CFSE; $5 \mu \mathrm{M}$; \#C34554; Invitrogen) cell staining was performed according to the manufacturer's instructions. To assess uptake in vitro, irradiated CFSE-labeled apoEG7 cells were cocultured with BMDCs at a 3:1 ratio for $3 \mathrm{~h}$ as previously described. ${ }^{36}$ Then, the mixed cells were stained with antibodies against CD11c (1:100; BD Biosciences) and LIVE/DEAD ${ }^{\text {TM }}$ Fixable Dead Cell Stain Kits (\#L34961; Thermo Fisher) according to the manufacturer's instructions and analyzed by flow cytometry.

Assays for oxidized mtDNA uptake

To measure $8-\mathrm{OHG}$ engulfment by $\mathrm{DCs}$, apoEG7 cells were cultured with BMDCs at a 1:1 ratio for $3 \mathrm{~h}$ in vitro. ${ }^{15}$ In vivo, mice were injected intraperitoneally with irradiated EG7 cells and housed for $18 \mathrm{~h}$. Cells in the peritoneal lavage fluid and BMDCs stimulated with EG7 cells were stained with goat anti-8-OHG (1:300; Abcam), Alexa Fluor 647-rabbit anti-goat lgG (1:1000; Thermo Fisher), hamster anti-CD11c (1:200; \#117301; BioLegend) and FITC-rabbit anti-hamster IgG (1:1000; Thermo Fisher) antibodies and observed under a Leica TCS SP5 confocal microscope after staining with DAPI. Moreover, cells in the peritoneal lavage fluid were stained with anti-CD11c, anti-CD45 and anti-8-OHG antibodies and analyzed by flow cytometry. BMDCs stimulated with EG7 cells were stained with anti-CD11c and anti-8-OHG antibodies and detected by flow cytometry.

Immunohistochemistry

Mice were challenged with $3 \times 10^{6}$ live EG7 cells, and EG7 tumors in mice were irradiated with $20 \mathrm{~Gy}$ on day 10 after tumor cell challenge. $^{37}$ Eighteen hours after irradiation, tumor tissue was obtained, snap frozen in liquid nitrogen, embedded in ornithine carbamyl transferase medium, and sectioned. The sections were fixed in acetone, briefly air dried, and blocked with $1 \%$ BSA. The sections were then stained with goat anti-8-OHG (1:300; Abcam) and Alexa Fluor 647-rabbit anti-goat IgG (1:1000; Thermo Fisher) antibodies and observed under a Leica TCS SP5 confocal microscope.
Western blot assay

Mitochondrial DNA isolated from EG7 cells was suspended in sterile $\mathrm{H}_{2} \mathrm{O}$ and treated with $75 \mathrm{~Gy}$ X-ray irradiation to generate oxidized mtDNA. ${ }^{24}$ BMDCs derived from WT mice were stimulated with $5 \mu \mathrm{g} / \mathrm{ml} \mathrm{mtDNA}$ after direct irradiation or $25 \mu \mathrm{g} / \mathrm{ml}$ DMXAA (5,6-dimethylxanthenone-4-acetic acid; Sigma; positive control $)^{38}$ for $3 \mathrm{~h}$. Proteins were extracted with radioimmunoprecipitation assay buffer (RIPA; Beyotime) containing proteinase inhibitors (Millipore) and phosphatase inhibitors (KeyGen). Briefly, $30 \mu \mathrm{g}$ of protein was electrophoresed in $12.5 \%$ SDS-PAGE gels and transferred to Immobilon-FL membranes (Millipore). The blots were incubated with antibodies specific for PTBK1 (Ser172), total TBK1, pIRF3 (Ser396), total IRF3 (Cell Signaling Technology) and $\beta$ actin (Zen BioScience). Quantitative analysis was performed with ImageJ software.

\section{Measurement of in vitro IFN- $\beta$ expression}

Mitochondrial DNA derived from untreated EG7 cells was irradiated with $75 \mathrm{~Gy}$. BMDCs were stimulated with irradiated mtDNA or nonirradiated mtDNA for $18 \mathrm{~h}$ in the presence of lipofectamine (Invitrogen). ${ }^{39}$ Mitochondrial DNA was complexed with Lipofectamine 2000 at a ratio of $12.5 \mu \mathrm{l}$ of lipofectamine to $2.5 \mu \mathrm{g}$ of mtDNA. Cells in 6-well plates were stimulated with $2.5 \mu \mathrm{g}$ of mtDNA. After an incubation, supernatants were collected, and IFN- $\beta$ concentrations were measured using the VeriKine ${ }^{\mathrm{TM}}$ Mouse IFN Beta ELISA Kit (\#42400; PBL Assay Science) in accordance with the manufacturer's instructions.

\section{OT-I T cell priming}

For in vitro priming, ${ }^{15,36}$ lymphocytes were isolated from the spleen of OT-I mice using mouse lymphocyte separation medium according to the manufacturer's instructions (\#7211011; DAKEWE). $\mathrm{CD}^{+} \mathrm{T}$ cells were magnetically sorted using a mouse $\mathrm{CD}^{+} \mathrm{T}$ cell isolation kit (\#19853; STEMCELL) in accordance with the manufacturer's instructions. BMDCs derived from WT or STING-deficient mice were cultured with irradiated EG7 cells for $18 \mathrm{~h}$ (DCs:EG7 cells $=1: 1$ ), and then $C D 11 c^{+}$BMDCs were isolated using a mouse CD11c-positive selection kit (\#18758; STEMCELL) according to the manufacturer's instructions. The sorted cell purity was confirmed by flow cytometry and found to be greater than $90 \%$. In addition to irradiation, EG7 cells were also treated with four freeze-thaw cycles using liquid nitrogen and a $37^{\circ} \mathrm{C}$ water bath. OT-I CD8 ${ }^{+}$ T cells labeled with CFSE $(5 \mu \mathrm{M})$ were seeded in 96 -well plates $\left(10^{5}\right.$ cells/well) together with CD11 $\mathrm{c}^{+}$BMDCs (DCs:CD8 ${ }^{+}$T cells $=1: 5$ and 1:10). Cocultures were performed for 3 days in triplicate. CFSE dilution in the $\mathrm{CD}^{+} \mathrm{T}$ cells was determined by flow cytometry.

\section{Flow cytometry analysis}

For cell-surface staining, cells were stained with antibodies (antiCD11C, anti-CD45, anti-MHC-II, and anti-CD8) for 30 min in PBS at $4{ }^{\circ} \mathrm{C}$ in the dark and then washed twice with PBS. For intracellular staining, cells were fixed in a $2 \%$ paraformaldehyde solution for 20 min at room temperature, permeabilized using 1\% Triton X-100 for $30 \mathrm{~min}$ at $4{ }^{\circ} \mathrm{C}$ and washed with PBS. Then, the cells were stained with intracellular antibodies (anti-caspase-3, anti-CD8, antiIFN- $\beta$, and anti-8-OHG) overnight at $4{ }^{\circ} \mathrm{C}$. Data acquisition was performed on a FACSAria III flow cytometer (BD Biosciences), and the results were analyzed using FlowJo software. Further gating adjustments were made based on fluorescence minus one controls.

\section{Immunofluorescence assay}

First, for cell-surface immunofluorescence staining, cells were stained with antibodies (hamster anti-CD11c) for 30 min in PBS at $4{ }^{\circ} \mathrm{C}$ and then washed twice with PBS. Second, for intracellular staining, cells were fixed in a $2 \%$ paraformaldehyde solution for $20 \mathrm{~min}$ at room temperature, blocked with $1 \%$ BSA for $30 \mathrm{~min}$ at $37^{\circ} \mathrm{C}$ and permeabilized using $1 \%$ Triton $\mathrm{X}-100$ for 30 min at $4{ }^{\circ} \mathrm{C}$. Then, the cells were stained with intracellular antibodies (anti- 
a

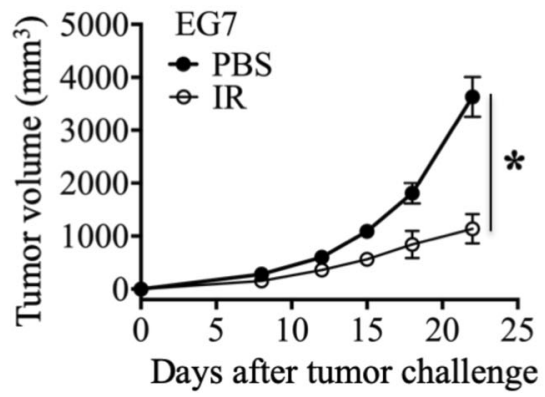

b

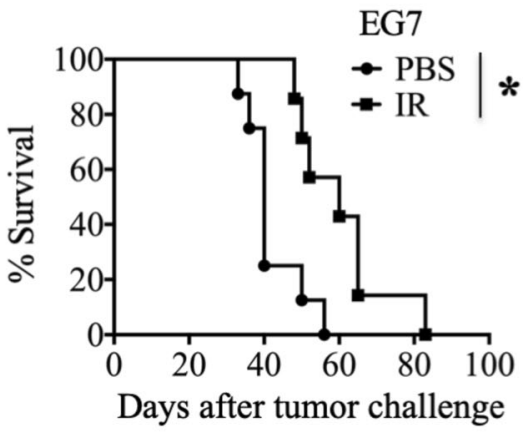

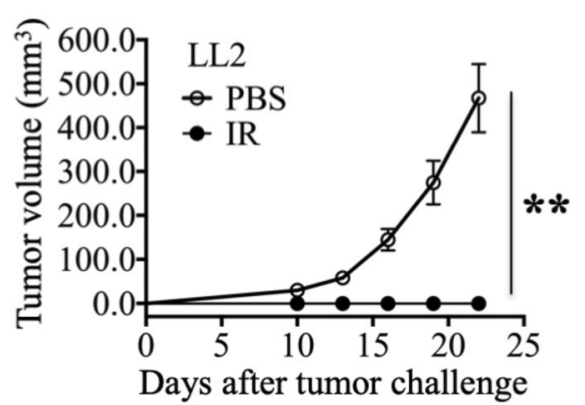

d

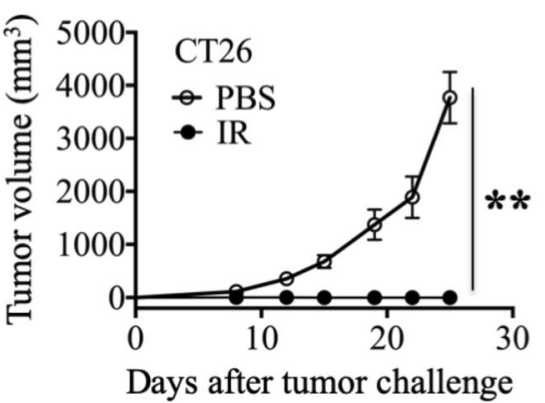

Fig. 1 Irradiated immunogenic tumor cell vaccine mediates protective antitumor effects. a Growth of subcutaneous EG7 tumors in C57BL/6 mice after preventive vaccination with irradiated EG7 tumor cells (IR) or PBS. b Percentage survival of EG7 tumor model mice. c, d Growth of subcutaneous LL2 tumors in C57BL/6 mice (c) and CT26 tumor in BALB/c mice (d) after preventive vaccination in the LL2 and CT26 models. Representative data are shown for three (a-d) experiments conducted with $10(\mathbf{a}, \mathbf{b})$ or $6-8(\mathbf{c}, \mathbf{d})$ mice per group. Data are represented as the mean \pm SEM. ${ }^{*} p<0.05,{ }^{* *} p<0.01$ (Student's $t$-test)

caspase- 3 and anti-8-OHG) overnight at $4{ }^{\circ} \mathrm{C}$. Finally, after staining with a secondary antibody and DAPI, the cells were observed under a Leica TCS SP5 confocal microscope.

Statistical analysis

Experiments were repeated three times. Data were analyzed using Prism 8.0 software (GraphPad Prism) and are presented as the mean \pm SEM. Statistical significance was assessed using two-tailed, unpaired Student's $t$-tests. Survival data were analyzed using the log-rank test. $p<0.05$ was considered significant.

\section{RESULTS}

Irradiated immunogenic tumor cell vaccine mediates a protective antitumor response

To investigate protective antitumor immunity, we immunized mice with an X-ray-irradiated immunogenic tumor cell vaccine or PBS on days 0,14 , and 21 and challenged them with tumor cells on day 7 after the third immunization. Tumor growth inhibition was determined by measuring tumor size. The irradiated immunogenic tumor cell vaccine demonstrated apparent and persistent protection against tumor growth in different tumor models established with three different mouse strains (Fig. 1a-d), including OVAexpressing mouse thymoma cells (EG7), mouse Lewis lung carcinoma cells (LL2) and mouse colon cancer cells (CT26). In the EG7 T cell lymphoma model, the survival time of the vaccine group was significantly longer than that of the PBS group (Fig. 1b). In addition, in the CT26 and LL2 models, the percentages of tumorfree mice in the irradiated tumor cell vaccine group was $100 \%$, but the group immunized with PBS had $100 \%$ tumor-bearing mice (Fig. 1c, d). However, the potential mechanisms by which ionizing radiation enhances the antitumor response of the irradiated tumor cell vaccine remain largely unknown. We hypothesize that the oxidized mtDNA-STING pathway plays an important role in the enhanced immunogenicity of irradiated cancer cell vaccines.
Irradiation induces cell apoptosis, and the antitumor effect does not correlate with mtDNA leakage from irradiated cells Intracellular molecules secreted, released and/or exposed by dying, injured or stressed cells, which are referred to as DAMPs, act as a danger signals to alert the immune system to abnormal cell injury or death. ${ }^{19}$ Our laboratory ${ }^{32}$ demonstrated that cell necrosis could be induced by cationic nanocarriers and the resulting leakage of mtDNA activated an innate immune response in vivo. It is conceivable that the free mtDNA released from irradiated dead cells as a DAMP is associated with the antitumor response induced by an irradiated cell vaccine. To test this hypothesis, we first examined tumor cell death induced by irradiation. EG7 cells treated with $75 \mathrm{~Gy}$ X-ray irradiation exhibited morphological changes characteristic of necrotic cells (cytoplasmic swelling, indicated by black arrow) and apoptotic cells (cell fragments, indicated by yellow arrow) (Fig. 2a). The treatment also led to an apparent increase in the number of necrotic cells and apoptotic cells, as detected by flow cytometry with Annexin-V and PI staining (Fig. 2b). PI-positive or Annexin-V-positive regions, which are recognized as dead cells, markedly increased from 4.2-20.3\% in vitro and from 5.2 to $10.6 \%$ in the peritoneal lavage fluid, as detected $24 \mathrm{~h}$ after X-ray treatment in the EG7 model (Fig. $2 \mathrm{c}$ ). Irradiation could also mediate cell death in an in vitro CT26 model, as determined by flow cytometry (Fig. 2d).

Next, we detected mtDNA leakage into the culture medium supernatant of irradiated EG7 cells by real-time quantitative PCR. Irradiation increased the concentration of free mtDNA (Fig. 2e). Release of mtDNA into the peritoneal lavage fluid was also confirmed $24 \mathrm{~h}$ after the injection of irradiated EG7 cells, and DNase I could degrade free mtDNA (Fig. 2f). Furthermore, we hypothesized that the X-ray dose affects the amount of mtDNA released from dead cells, subsequently modulating the antitumor effects. Thus, an X-ray dose-escalation study to investigate the effect of the irradiated tumor cell vaccine was performed. However, no differences in tumor volume after immunization 

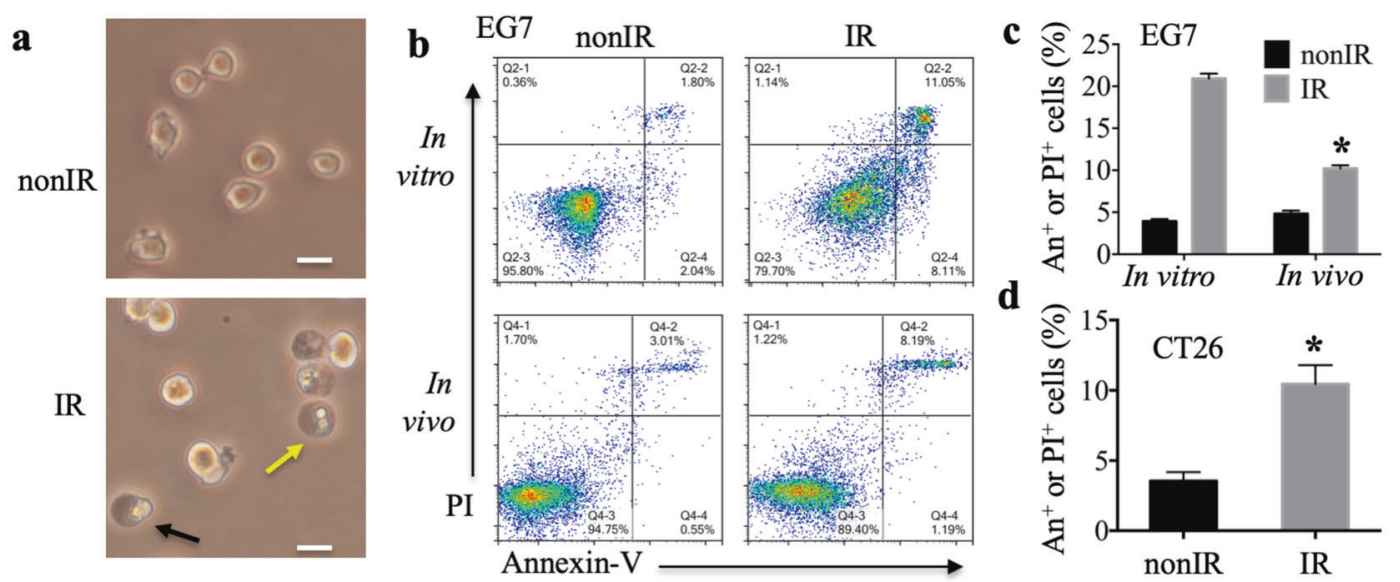

d

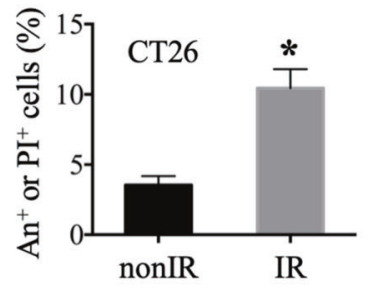

$\mathbf{e}$

f

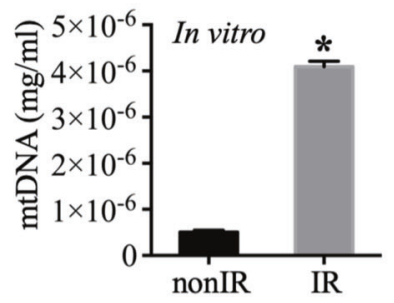

ह

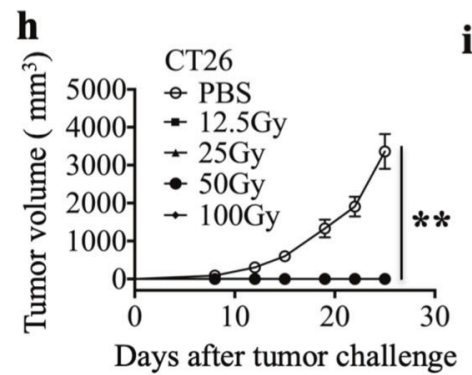

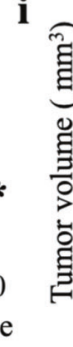

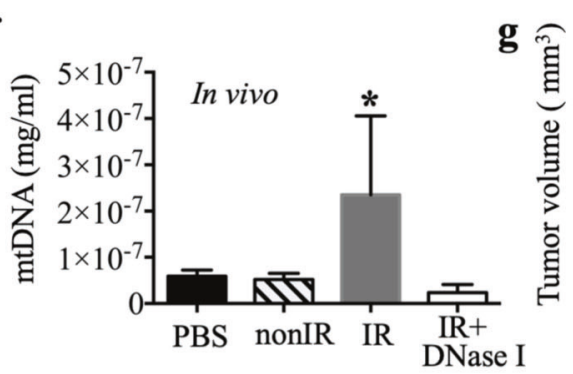

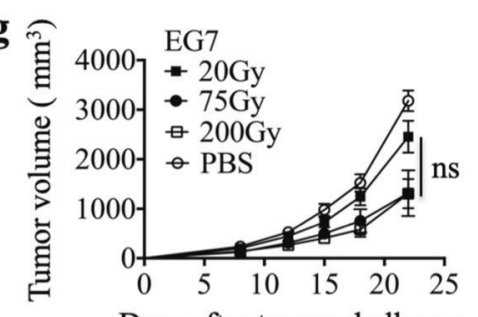

Days after tumor challenge
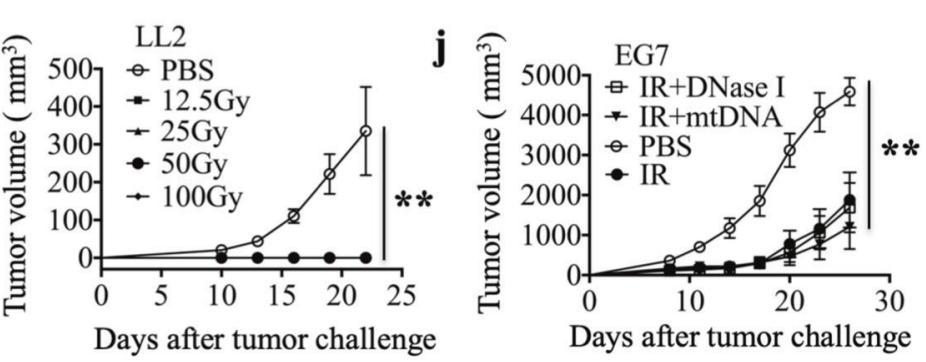
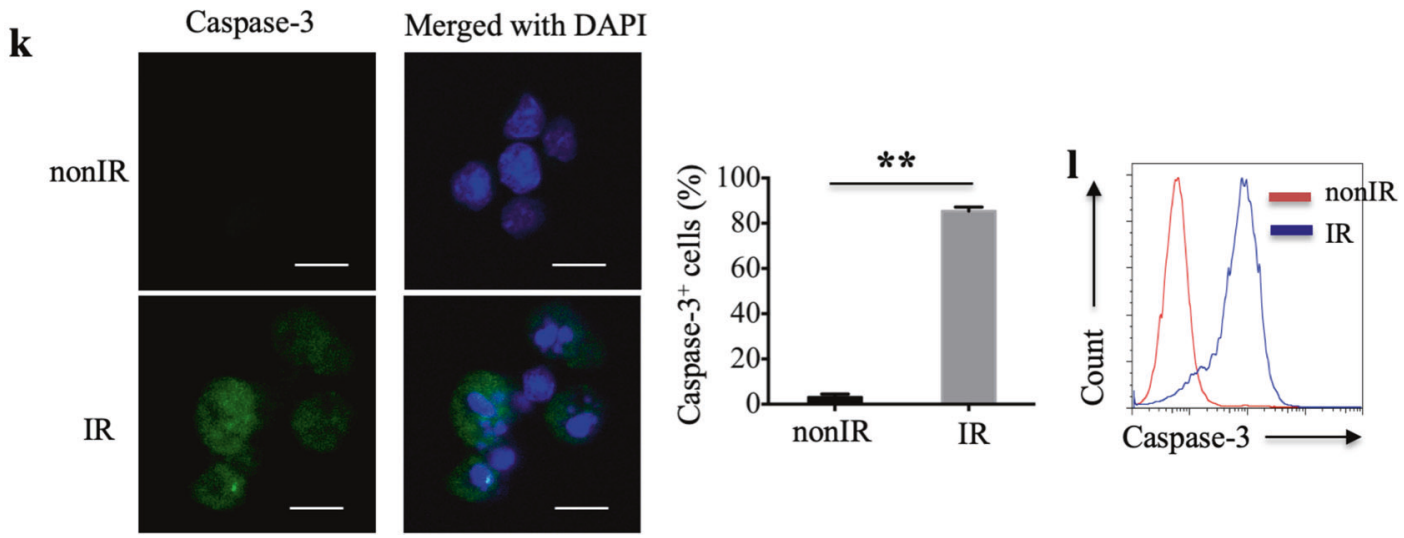

Fig. 2 Irradiation induces cell apoptosis, and the antitumor response induced by the irradiated tumor cell vaccine does not correlate with mtDNA leakage from irradiated cells. a The morphological changes in untreated EG7 cells (nonIR) and irradiated EG7 cells (IR); scale bar, $20 \mu \mathrm{m}$. b A representative experiment for the detection of untreated EG7 cells and dead EG7 cells induced by irradiation after $24 \mathrm{~h}$ in vitro or in vivo, as determined by flow cytometry analysis of Annexin-V and PI staining. c, $\mathbf{d}$ Percentages of Annexin- $\mathrm{V}^{+}$or $\mathrm{PI}^{+}$cells in all cells in the EG7 model (c) and CT26 model (d). e, f Quantity of free mtDNA in supernatants derived from untreated EG7 cells or irradiated EG7 cells in vitro (e) and in the peritoneal lavage fluid of mice treated intraperitoneally with PBS, untreated EG7 cells, irradiated EG7 cells or irradiated EG7 cells with DNase I (f). g Growth of subcutaneous EG7 tumors in C57BL/6 mice after preventive vaccination with EG7 cells irradiated with different X-ray doses or PBS. h, i Growth of subcutaneous CT26 tumors in BALB/c mice (h) and LL2 tumor in C57BL/6 mice (i) after preventive vaccination with tumor cells irradiated with different X-ray doses or PBS. $\mathbf{j}$ Preventive vaccination with irradiated EG7 cells combined with mtDNA derived from untreated EG7 cells or treated with DNase I. k A representative immunofluorescence experiment with staining for Caspase-3 (green) and cell nuclei (blue) in untreated EG7 cells and irradiated EG7 cells; scale bar, $10 \mu \mathrm{m}$. The percentage of Caspase- $3^{+}$EG7 cells in total DAPI-positive nuclei was calculated over ten $600 \times$ fields. I Caspase-3 detected by flow cytometry. Representative data are shown for three (a-I) experiments conducted with 6-9 (g-j) mice per group. Data are represented as the mean \pm SEM. ${ }^{*} p<0.05,{ }^{* *} p<0.01$, ns not significant (Student's $t$-test) 
$\mathbf{a}$

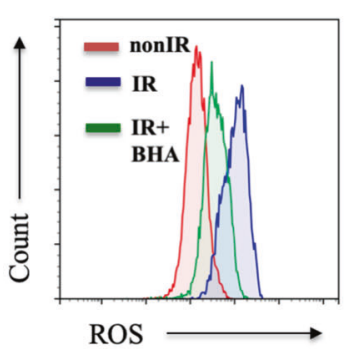

b

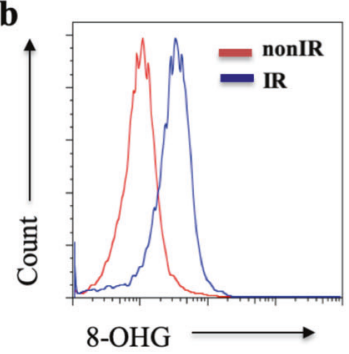

c

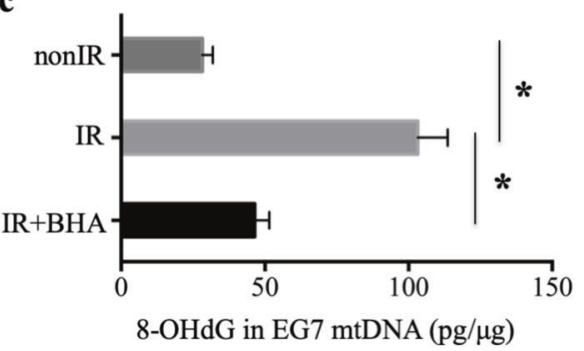

d

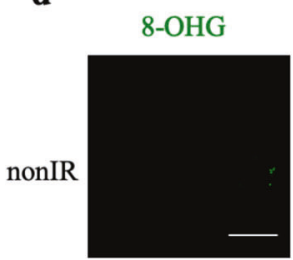

MitoTracker
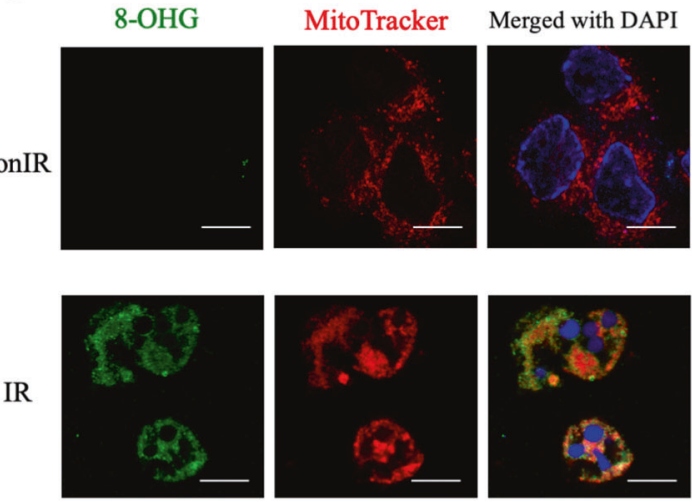

$\mathbf{e}$
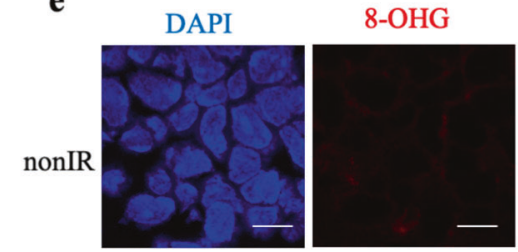

Merged with DAPI

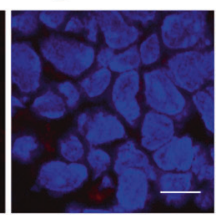

IR

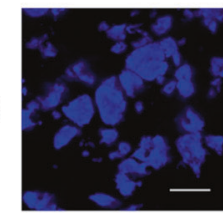

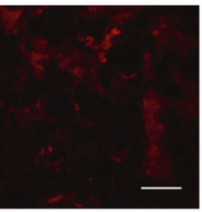

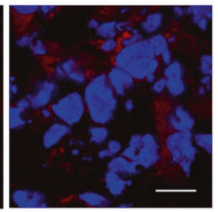

Fig. 3 Oxidative damage to mtDNA after irradiation. a EG7 cells were treated with X-ray irradiation in the presence of the ROS scavenger BHA (BHA-plus EG7). ROS production in EG7 cells was measured using the dye DCF. b 8-OHG in EG7 cells was detected by flow cytometry. c The concentration of 8-OHdG $(\mathrm{pg})$ in mtDNA $(\mu \mathrm{g})$ isolated from EG7 cells was measured with a competitive 8-OHdG EIA kit. d Immunofluorescence staining with an anti-8-OHG antibody (green), MitoTracker (red), and DAPI (cell nuclei, blue) was performed with untreated EG7 cells and apoEG7 cells; scale bar, $10 \mu \mathrm{m}$. e Immunofluorescence staining for 8-OHG (red) and cell nuclei (DAPI, blue) in untreated EG7 tumor sections and irradiated EG7 tumor sections was performed; scale bar, $10 \mu \mathrm{m}$. Data from three independent experiments were pooled and are shown as the means \pm SEM. ${ }^{*} p<0.05$ (Student's $t$-test)

with irradiated tumor cell vaccines created with different X-ray doses were noted in the three tumor models (Fig. $2 \mathrm{~g}-\mathrm{i}$ ). All the tumor cell vaccines irradiated with a dose between 12.5 Gy and $100 \mathrm{~Gy}$ had $100 \%$ tumor-free mice in the CT26 and LL2 models (Fig. 2h, i). In the EG7 model, although it appeared that 20 Gy was not different from the other doses, the difference was not statistically significant $(p>0.05)$ (Fig. $2 g$ ). Mice were immunized subcutaneously in the left side with the irradiated tumor cell vaccine, and we measured the tumors in the right flank of the immunized mice after tumor challenge. However, tumors were noted in the left side from day 12 to day 22 in 3/9 mice after immunization with the irradiated EG7 cell vaccine created with 20 Gy, which did not occur with the vaccines created with 75 or 200 Gy (data not shown). This result demonstrates that irradiation with $20 \mathrm{~Gy}$ is insufficient to inactivate EG7 tumor cells, which increases the tumor burden and may affect the antitumor response induced by the irradiated EG7 vaccine (Fig. $2 \mathrm{~g}$ ). Moreover, treatment of mice with DNase I after immunization failed to prevent the antitumor activity induced by the irradiated vaccine (Fig. 2j). The addition of purified mtDNA derived from untreated EG7 cells did not potentiate the antitumor activity induced by the irradiated tumor cell vaccine (Fig. 2j). We deduced that free mtDNA was minimally released from cells undergoing apoptosis, which is the major type of cell death occurring after irradiation because preservation of the cell membrane during apoptosis results in limited stimulation of host immune cells. The induction of cell apoptosis by radiotherapy was further confirmed in the present study. Apoptotic markers of caspase activation (caspase-3-p20 immunoreactivity) and apoptotic fragmented nuclei appeared in the cytoplasm of irradiated cells after X-ray irradiation (Fig. 2k). The percentage of caspase-3-positive EG7 cells among the total population of cells with a DAPI-positive nucleus induced $24 \mathrm{~h}$ after irradiation was increased in the irradiated group compared with the nonirradiated group (Fig. 2k), and this finding was also evident by flow cytometry (Fig. 2l). One previous study ${ }^{15}$ demonstrated that DNA from irradiated tumor cells is sensed by host cGAS during a cell-cell contact-mediated process and DCs do not engulf free DNA fragments, which was confirmed by the application of a physical barrier, an actin polymerization inhibitor and DNase I. Therefore, it is possible that mtDNA leakage from irradiated cells does not correlate with the antitumor response elicited by the irradiated cell vaccine.

\section{Oxidative damage of mtDNA after irradiation}

To explore the role of oxidized mtDNA in the activation of the antitumor effect by the irradiated tumor cell vaccine, oxidative damage experiments were performed. ROS production was markedly increased after X-ray irradiation (Fig. 3a). Butylated hydroxyanisole (BHA), a ROS scavenger, ${ }^{29,40}$ demonstrated the ability to decrease the ROS levels induced by irradiation in our study (Fig. 3a). ROS are highly reactive with DNA and induce distinct DNA modifications. The oxidation of guanine to 8-OHG (8hydroxyguanosine), a hallmark of oxidative DNA damage, ${ }^{41}$ was increased in EG7 cells treated with $75 \mathrm{~Gy} X$-ray irradiation after $18 \mathrm{~h}$ (apoptotic EG7, apoEG7 cells) compared with untreated EG7 cells, as detected by flow cytometry (Fig. 3b). Moreover, the 8-hydroxy-2deoxyguanosine (8-OHdG) content in mtDNA was enhanced in irradiated apoEG7 cells but was decreased by BHA, as measured using an 8-OHdG EIA kit (Fig. 3c). Unlike nuclear DNA (nDNA), mtDNA is not protected by histones. Therefore, the proofreading capacity is limited, and mtDNA is more easily attacked by ROS. ${ }^{22}$ Indeed, apoEG7 cells exhibited intense labeling in the peripheral nuclear region with minimal 8-OHG in the central area in our study (Fig. 3d), which was also demonstrated in irradiated tumor sections from an in vivo experiment (Fig. 3e). Moreover, to label mitochondria, cells were incubated with MitoTracker probes, the 
signal for oxidized 8-OHG colocalized with MitoTracker fluorescence, and the Pearson's correlation coefficient was +0.60 (a perfect linear correlation is +1 ) (Fig. 3d), confirming that most 8OHG oxidation induced by X-ray irradiation occurred in mitochondrial DNA rather than in chromosomal DNA.

Oxidized mtDNA from irradiated cells gains access to the cytoplasm of DCs

To investigate whether oxidative mtDNA from irradiated tumor cells is delivered to the cytosol of DCs, irradiated CFSE-labeled apoEG7 cells were cocultured with BMDCs (bone marrow-derived DCs) for $3 \mathrm{~h}$. We found that BMDCs engulfed more irradiated CFSElabeled apoEG7 cells than nonirradiated EG7 cells in vitro (Fig. 4a and supplementary Fig. S1a). We also observed that approximately $45 \%$ of $\mathrm{CD}_{11 \mathrm{c}^{+}} \mathrm{DCs}$ showed positive staining for oxidized 8-OHG derived from irradiated EG7 tumor cells, whereas controls showed only $4-5 \%$ positive staining, as assessed by flow cytometry (Fig. 4b). Furthermore, we confirmed the result with an independent protocol for mtDNA depletion with dideoxycytidine (ddC), an inhibitor of mtDNA polymerase $\gamma$ that does not affect the functions of nuclear DNA polymerases. ${ }^{42,43}$ We cultured EG7 tumor cells with dideoxycytidine (ddC), which efficiently depleted mtDNA from EG7 cells (Fig. 4c) and decreased the amounts of oxidized mtDNA ingested by DCs, as detected by flow cytometry (Fig. 4d). Moreover, BMDCs were cultured with apoEG7 cells for 3 $\mathrm{h}$, and genomic DNA (gDNA) isolated from purified CD11c ${ }^{+}$ BMDCs was evaluated using an $8-O H d G$ EIA kit. Similarly, higher levels of 8-OHdG in gDNA were noted in BMDCs cocultured with apoEG7 cells than in those cocultured with nonirradiated EG7 cells or BHA-plus apoEG7 cells (Fig. 4e). To further confirm whether 8OHG derived from apoEG7 cell mtDNA gains access to the cytoplasm of DCs, BMDCs were cultured with apoEG7 cells for $3 \mathrm{~h}$ and evaluated by immunocytochemistry. As noted for the irradiation group, oxidized mtDNA derived from irradiated EG7 cells was detected in the cytoplasm of DCs (Fig. 4f).

Moreover, $18 \mathrm{~h}$ after subcutaneous immunization with irradiated EG7 cells, elevated amounts of $\mathrm{CD}_{4} 5^{+} \mathrm{CD} 11 \mathrm{C}^{+} \mathrm{MHC}-\mathrm{II}^{+} \mathrm{DCs}$ among CD45 ${ }^{+}$leukocytes were found in the mouse skin (Fig. 5a). The ROS scavenger BHA reduced the percentage of DCs recruited to the skin (Fig. 5a). To further assess whether oxidized mtDNA was transferred to DCs in vivo, mice were treated intraperitoneally with irradiated EG7 cells and housed for $18 \mathrm{~h}$. The percentage of $\mathrm{CD}_{4}{ }^{+} \mathrm{CD} 11 \mathrm{c}^{+} 8-\mathrm{OHG}^{+}$cells in $\mathrm{CD} 45^{+} \mathrm{CD} 11 \mathrm{c}^{+} \mathrm{DCs}$ in the peritoneal lavage fluid was markedly increased after treatment with irradiated EG7 cells compared with treatment with nonirradiated EG7 cells or PBS (Fig. 5b and Supplementary Fig. S1b). Moreover, both BHA and ddC decreased the amount of oxidized mtDNA ingested by DCs in vivo (Fig. 5b). Furthermore, as observed for the irradiation group, oxidized mtDNA derived from irradiated EG7 cells could be detected in the cytoplasm of DCs in vivo by immunocytochemistry (Fig. 5c). Collectively, these in vitro and in vivo data suggest that oxidized mtDNA gains access to the cytosol of DCs.

Oxidized mtDNA activates the STING pathway in DCs, leading to $\mathrm{CD}^{+} \mathrm{T}$ cell proliferation

Oxidized DNA modified with 8-OHG after UV irradiation is resistant to cytosolic exonuclease TREX-1-mediated degradation, and STING-dependent signaling but not TLR9 signaling mediates the type I IFN response triggered by UV irradiation-damaged DNA. ${ }^{24}$ To validate the role of oxidized mtDNA in STING signaling activation, mtDNA isolated from EG7 cells and directly exposed to $X$-ray irradiation was used to stimulate BMDCs. TANK-binding kinase 1 (TBK1) phosphorylation and IRF3 (IFN regulatory factor 3) phosphorylation in BMDCs were detected by immunoblot analysis. We observed increased phosphorylation of TBK1 and IRF3 in BMDCs stimulated with irradiated oxidized mtDNA compared with those stimulated with nonirradiated mtDNA. The amount of each protein was normalized to that of the $\beta$-actin loading control, and the ratio of phosphorylated protein to total protein was quantified (pTBK1/TBK1: IR [0.414] versus nonIR [0.139], versus unstimulated DCs [0.104], $p<0.01 ;$ pIRF3/IRF3: IR [0.221] versus nonIR [0.185], versus unstimulated DCs [0.138], $p<0.05$ ) (Fig. 6a). STING is a major regulator of type I IFN induction by intracellular exogenous pathogen-derived DNA in a TLR-independent manner. ${ }^{44,45}$ To test whether the oxidized mtDNA-IRF3-STING pathway is responsible for type I IFN induction following irradiation, an in vitro experiment utilizing $\mathrm{mtDNA}$ after exposure to direct irradiation or nonirradiated mtDNA combined with lipofectamine showed that the irradiated mtDNA provoked greater IFN- $\beta$ production by BMDCs (Fig. 6b). Moreover, we measured the percentage of BMDCs that demonstrated IFN- $\beta$ secretion after coculturing BMDCs with apoEG7 cells for $3 \mathrm{~h}$. There were more CD11 $\mathrm{c}^{+} \mathrm{IFN}-$ $\beta^{+}$cells among CD11 $\mathrm{c}^{+}$BMDCs in the irradiation groups than the nonirradiated EG7 cell groups, as assessed by flow cytometry (Fig. 6c). We also measured the percentage of $\mathrm{CD} 45^{+} \mathrm{CD} 11 \mathrm{c}^{+} \mathrm{IFN}-$ $\beta^{+}$DCs in the skin $18 \mathrm{~h}$ after subcutaneous immunization with irradiated EG7 cells. As shown in Fig. 5a, relatively high numbers of $\mathrm{CD} 45^{+} \mathrm{CD} 11 \mathrm{c}^{+} \mathrm{MHC}-\mathrm{II}^{+} \mathrm{DCs}$ and $\mathrm{CD} 45^{+} \mathrm{CD} 11 \mathrm{c}^{+} \mathrm{IFN}-\beta^{+} \mathrm{DCs}$ among all $\mathrm{CD} 45^{+}$cells were recruited into the mouse skin (Fig. $6 \mathrm{~d}$ ).

To map whether the functional capability of DCs to crosspresent antigen is potentiated by stimulation with irradiated tumor cells compared with stimulation with nonirradiated tumor cells, we treated EG7 cells with irradiation, freeze-thaw cycles or apoEG7 cells with the addition of BHA. EG7 cells were cultured with BMDCs derived from WT mice for $18 \mathrm{~h}$ (loaded BMDCs). Subsequently, sorted $C D 11 \mathrm{C}^{+}$BMDCs isolated from the loaded BMDCs were incubated with CFSE-stained purified $\mathrm{CD}^{+} \mathrm{T}$ cells derived from OT-I mice for 3 days. We estimated the proliferation of the $C D 8^{+} \mathrm{T}$ cells by measuring the percentage of CFSE ${ }^{\text {low }} \mathrm{CD} 8^{+}$ $\mathrm{T}$ cells among all $\mathrm{CD} 8^{+} \mathrm{T}$ cells by flow cytometry. BMDCs alone or BMDCs stimulated with a freeze-thaw lysate failed to induce $C D 8^{+}$ $T$ cell proliferation, but BMDCs treated with EG7 cells could induce the proliferation of OVA-specific CD8 ${ }^{+} T$ cells (Fig. 6e, $f$ and supplementary Fig. S2a). Furthermore, the proliferation induced by loaded BMDCs stimulated with irradiated EG7 (apoEG7) cells was increased compared with that induced by BMDCs treated with nonirradiated EG7 cells (Fig. 6e, f). In conclusion, oxidized mtDNA ingested by DCs activates the TBK1-IRF3-IFN- $\beta$ pathway in $\mathrm{DCs}$, leading to $\mathrm{CD}^{+} \mathrm{T}$ cell proliferation.

The oxidized mtDNA-STING pathway plays key roles in the enhanced antitumor response achieved with an irradiated cell vaccine

To further explore the role of oxidized mtDNA-STING signaling in the enhanced antitumor effect triggered by the irradiated cell vaccine, in an OT-I T cell priming experiment, we observed that the proliferation of $\mathrm{CD}^{+}$T cells induced by loaded BMDCs treated with apoEG7 cells with the addition of BHA was decreased compared with that induced by BMDCs stimulated with apoEG7 cells (Fig. 7a and Supplementary Fig. S2b). We also found that treatment of irradiated EG7 cells with the ROS scavenger BHA significantly impaired the irradiated EG7 cell vaccine-mediated antitumor effect (Fig. 7b), demonstrating that oxidative damage is important for the antitumor effect induced by the irradiated tumor cell vaccine. Furthermore, ddC, an inhibitor of mtDNA polymerase, $\gamma$, also had the ability to impair the proliferation of $\mathrm{CD}^{+} \mathrm{T}$ cells activated by irradiated EG7 cells (Fig. 7c and Supplementary Fig. S2c). In addition, the absence of host STING prevented the induction of $\mathrm{CD}^{+} \mathrm{T}$ cell proliferation activated by irradiated EG7 cells in the OT-I T cell priming experiment (STING is encoded by Tmem173) (Fig. 7d). Moreover, EG7 cells were implanted into the flanks of WT (wild-type) and STING-deficient mice after three protective immunizations with an X-ray-irradiated EG7 cell vaccine, and tumor growth was monitored. We again found that the absence of host STING significantly impaired the antitumor effect of the cell vaccine (Fig. 7e), demonstrating that 

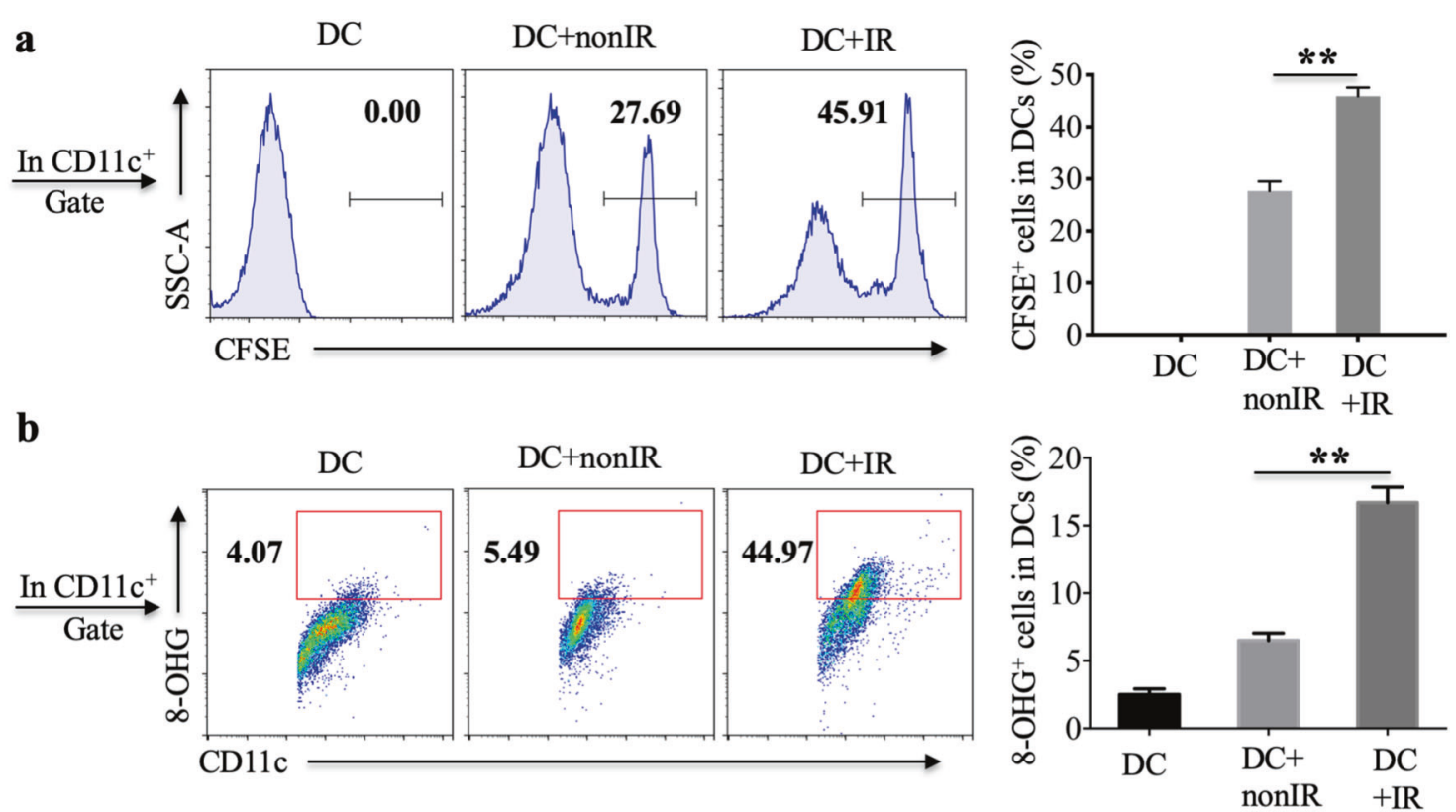

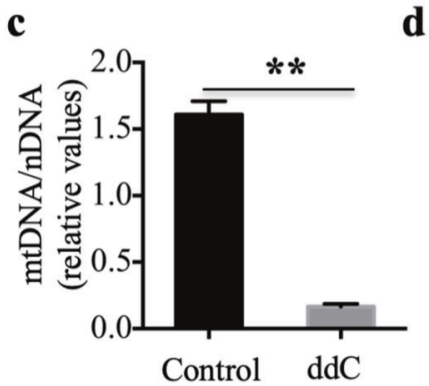

f
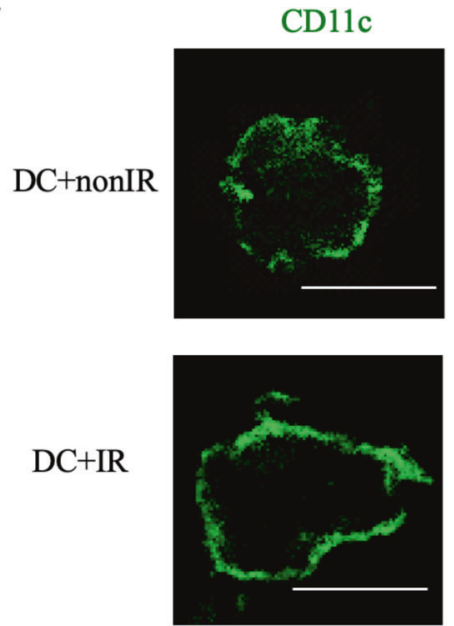

d

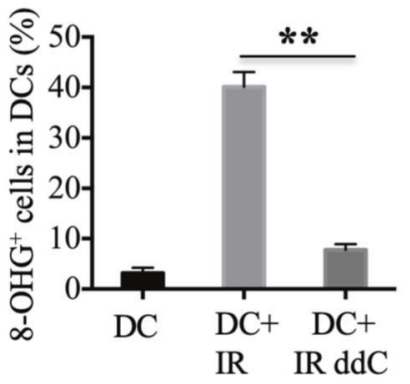

$\mathbf{e}$
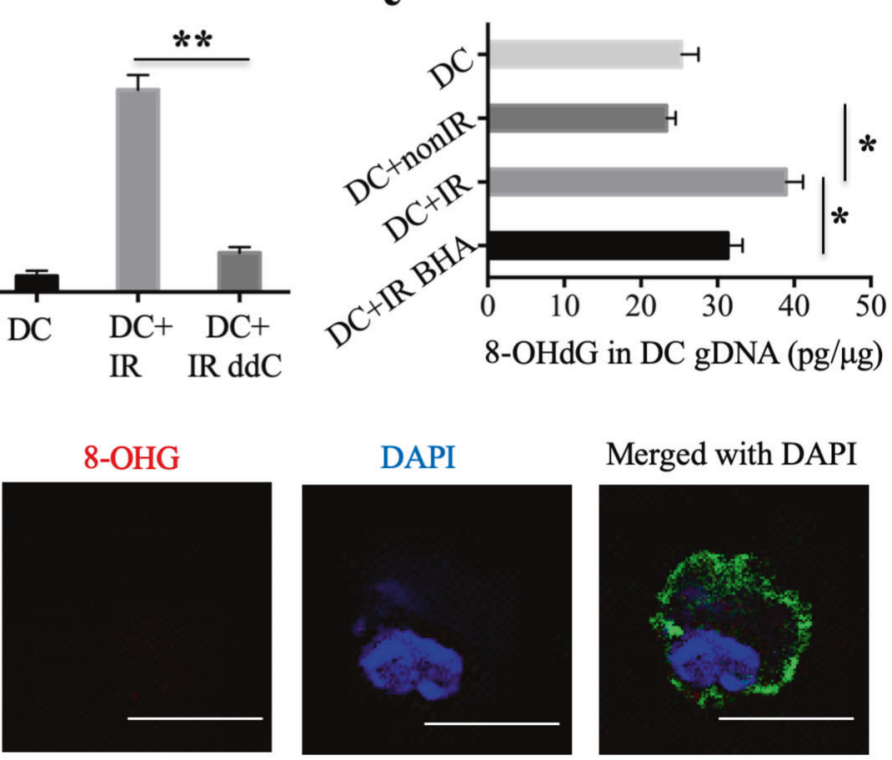

Merged with DAPI
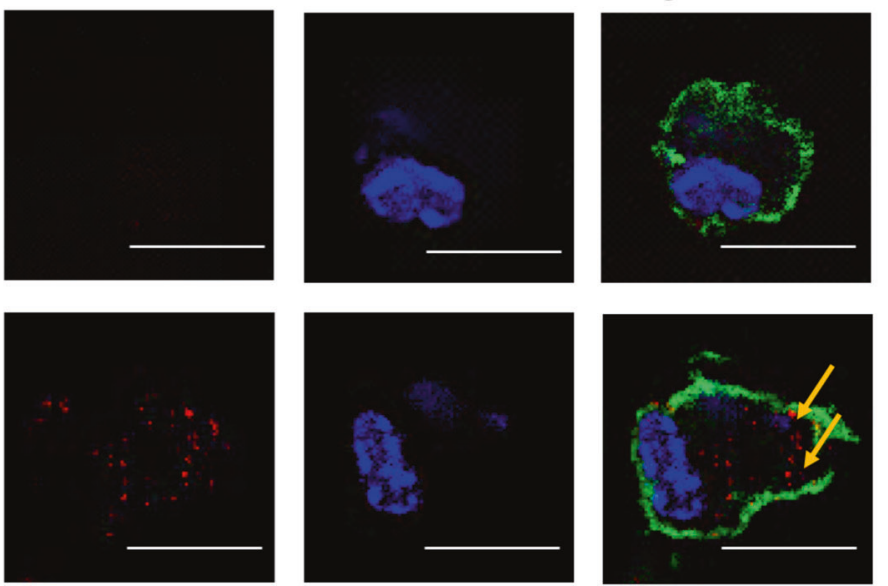

Fig. 4 Oxidized mtDNA of irradiated cells gains access to the cytoplasm of DCs in vitro. a BMDCs were cultured with nonirradiated CFSEstained EG7 cells or irradiated CFSE-stained apoEG7 cells for $3 \mathrm{~h}$ in vitro, subsequently labeled with an anti-CD11c antibody, and detected by flow cytometry. The percentages of $\mathrm{CD} 11 \mathrm{c}^{+} \mathrm{CFSE}^{+}$cells in $\mathrm{CD} 11 \mathrm{c}^{+} \mathrm{DCs}$ are shown. b BMDCs were cultured with untreated EG7 cells or apoEG7 cells for $3 \mathrm{~h}$ (stimulated DCs). The percentages of CD11 $\mathrm{c}^{+} 8-\mathrm{OHG}^{+}$cells in $\mathrm{CD} 11 \mathrm{c}^{+} \mathrm{DCs}$ were determined by flow cytometry. $\mathrm{c}$ EG7 cells were treated with dideoxycytidine $(\mathrm{ddC}, 150 \mu \mathrm{M})$ for 6 days (ddC-plus EG7). The ratio of mitochondrial DNA to nuclear DNA was measured by realtime PCR, and untreated EG7 cells were used as a control. d BMDCs were cultured with ddC-plus apoEG7 cells for $3 \mathrm{~h}$, subsequently labeled with anti-CD11c and anti-8-OHG antibodies and detected by flow cytometry. e Genomic DNA ( $\mu$ g) isolated from CD11 ${ }^{+}$cells purified from stimulated DCs was evaluated with the 8-OHdG (pg) EIA Kit. BMDCs were also cultured with BHA-plus apoEG7 cells for $3 \mathrm{~h}$. $\mathrm{f}$ Stimulated DCs were labeled to visualize CD11c (green), 8-OHG (red), and cell nuclei (DAPI, blue); scale bar, $10 \mu \mathrm{m}$. Data are representative of three experiments and presented as the mean \pm SEM. ${ }^{*} p<0.05,{ }^{* *} p<0.01$ (Student's $t$-test) 
a

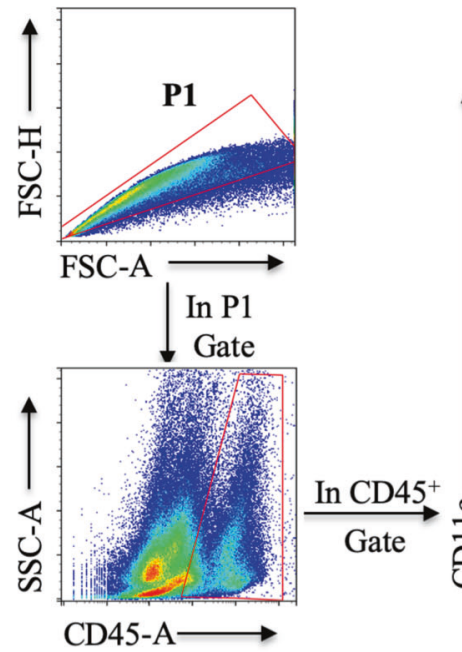

PBS

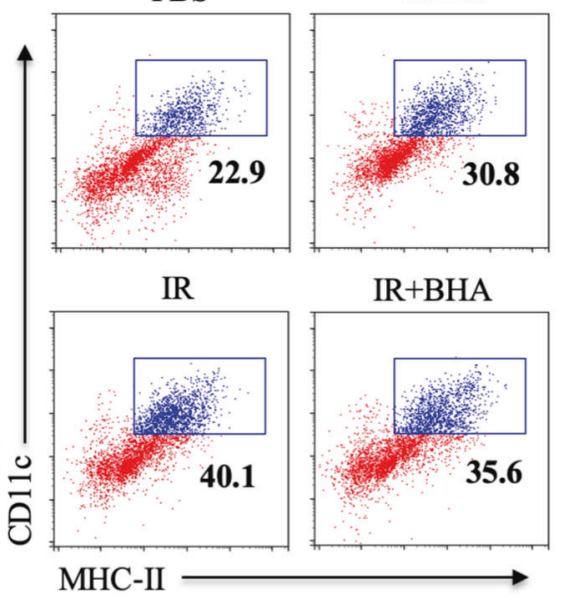

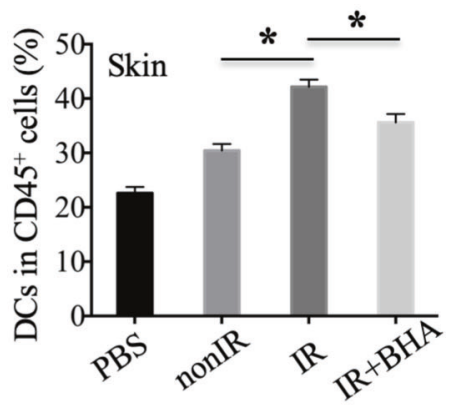

b
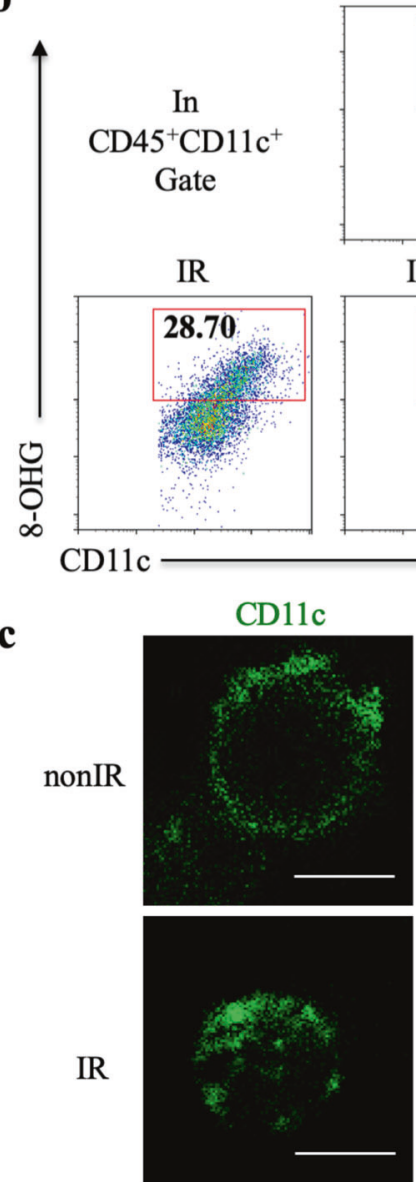

PBS

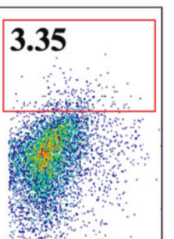

$\mathrm{IR}+\mathrm{BHA}$

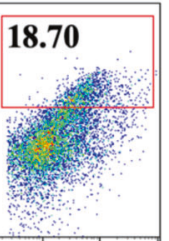

$8-\mathrm{OHG}$
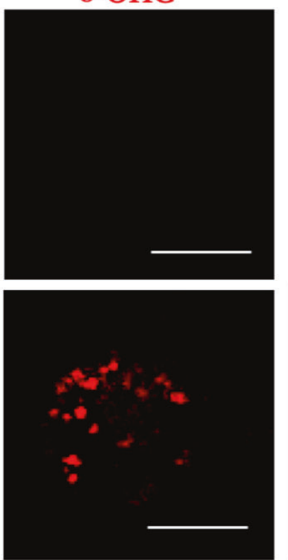

nonIR

4.24

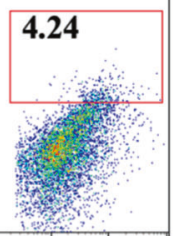

$\mathrm{IR}+\mathrm{ddC}$

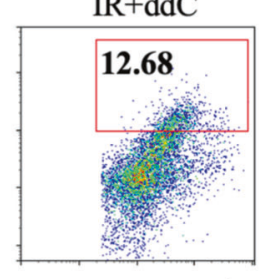

DAPI

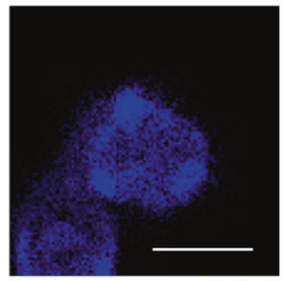

Merged with DAPI
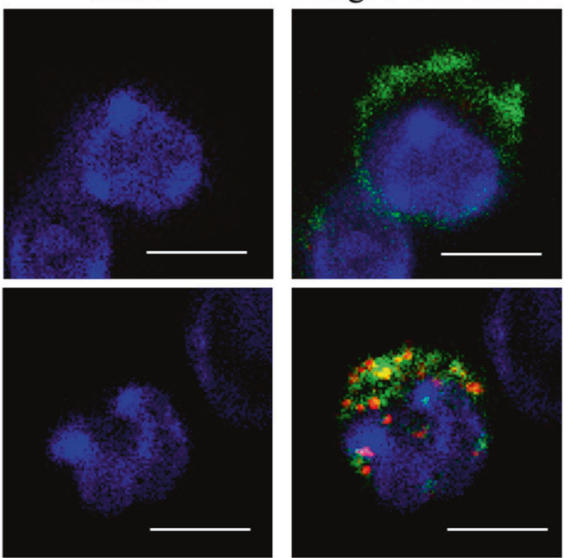

Fig. 5 Oxidized mtDNA derived from irradiated tumor cells is transferred to host DCs in vivo. a Skin cells isolated from mice $18 \mathrm{~h}$ after subcutaneous injection of PBS, untreated EG7 cells, irradiated EG7 cells or BHA-plus irradiated EG7 cells were analyzed by flow cytometry. The percentages of $\mathrm{CD}_{4} 5^{+} \mathrm{CD} 11 \mathrm{c}^{+} \mathrm{MHCll}^{+} \mathrm{DCs}$ among $\mathrm{CD} 45^{+}$cells in the skin are shown. $\mathbf{b}$ Cells in the peritoneal lavage fluid of mice treated intraperitoneally with PBS, untreated EG7 cells, irradiated EG7 cells, BHA-plus EG7 or ddC-plus EG7 (stimulated DCs in vivo) were labeled with anti-CD45, anti-CD11c and anti-8-OHG antibodies and assessed by flow cytometry $(n=3)$. c Stimulated DCs in vivo were labeled to visualize CD11c (green), 8-OHG (red) and cell nuclei (DAPI, blue); scale bar, $5 \mu \mathrm{m}$. Representative data are shown for three experiments conducted with 3-5 mice per group. Data are represented as the mean \pm SEM. ${ }^{*} p<0.05,{ }^{* *} p<0.01$ (Student's $t$-test) 

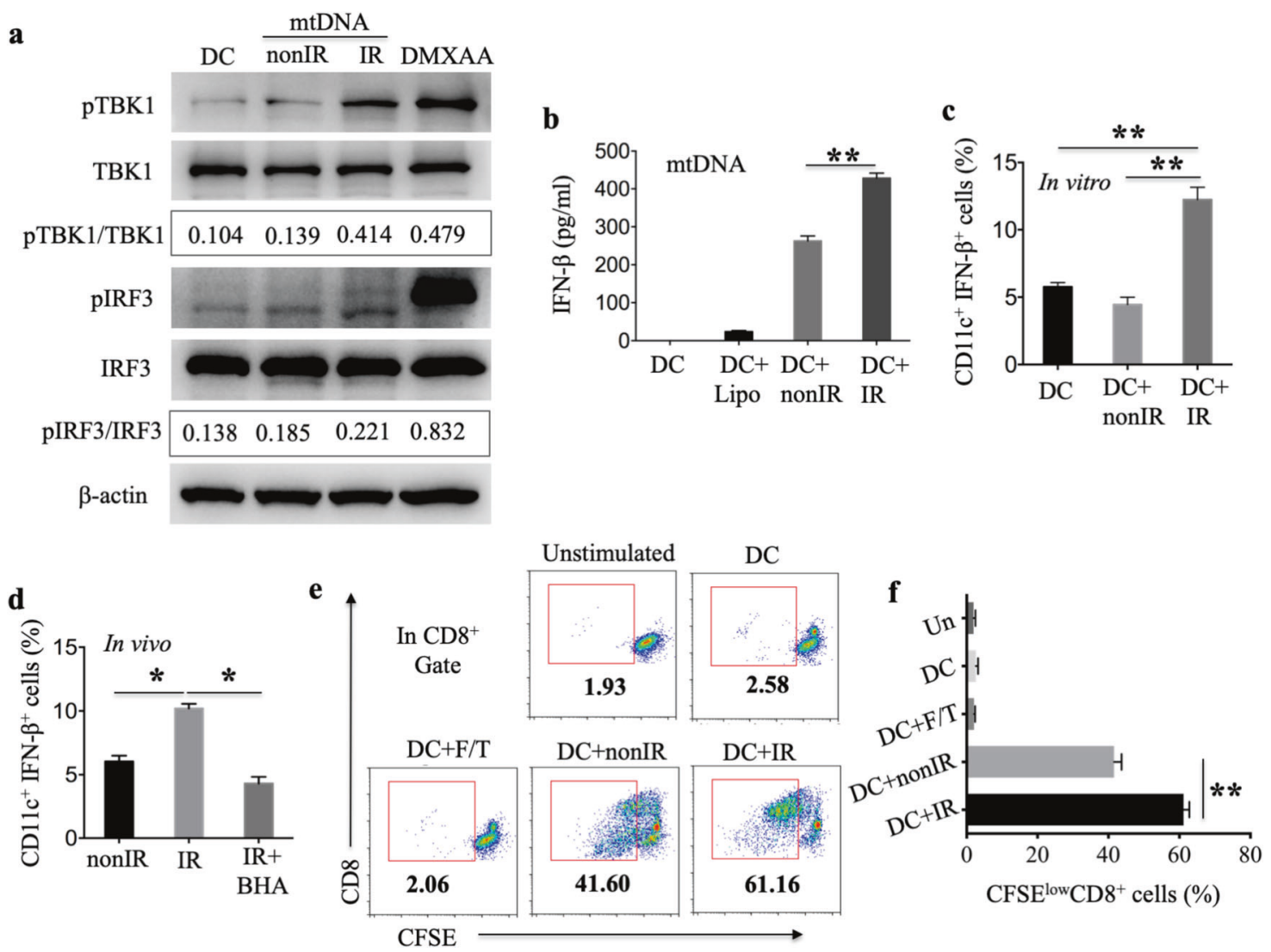

Fig. 6 Oxidized mtDNA ingested by DCs activates the TBK1-IRF3-IFN- $\beta$ pathway in DCs, which leads to CD8+ T cell proliferation. a Mitochondrial DNA isolated from EG7 cells was directly irradiated with X-rays. BMDCs were subsequently stimulated with mtDNA for $3 \mathrm{~h}$, and the amounts of pTBK1, total TBK1, pIRF3, total IRF3, and $\beta$-actin were measured by immunoblotting. DMXAA was used as a positive control. b BMDCs were stimulated with directly irradiated mtDNA for $18 \mathrm{~h}$ in the presence of lipofectamine, and the supernatants were collected to measure IFN- $\beta$ levels by ELISA. c BMDCs, which were cultured with untreated EG7 cells or apoEG7 cells for $3 \mathrm{~h}$, were labeled with anti-CD11c and anti-IFN- $\beta$ antibodies and evaluated by flow cytometry. The percentages of CD $11 c^{+}$IFN- $\beta^{+}$cells in CD11 ${ }^{+}$BMDCs are shown. d Skin cells collected as described in Fig. 5a were labeled with anti-CD11C, anti-CD45 and anti-IFN- $\beta$ antibodies and assessed by flow cytometry. The percentages of $C D 45^{+} \mathrm{CD} 11 \mathrm{c}^{+} \mathrm{IFN}-\beta^{+} \mathrm{DCs}$ among $\mathrm{CD} 45^{+}$cells are shown $(n=3)$. e, $\mathbf{f}$ EG7 cells treated with irradiation or freezethaw cycles $(F / T)$ were cultured with BMDCs from WT mice for $18 \mathrm{~h}$ (loaded DCs). Subsequently, CD11 $\mathrm{c}^{+}$cells purified from the loaded DCs were incubated with sorted CFSE-stained CD8 ${ }^{+} \mathrm{T}$ cells from OT-I mice (DCs:CD8 ${ }^{+} \mathrm{T}$ cells $=1: 5$ ). We estimated the proliferation of the CD8 ${ }^{+}$ $\mathrm{T}$ cells by measuring the percentages of CFSE ${ }^{\text {low }} \mathrm{CD}^{+} \mathrm{T}$ cells in $\mathrm{CD} 8^{+} \mathrm{T}$ cells by flow cytometry. Data are representative of three experiments and presented as the mean \pm SEM. ${ }^{*} p<0.05,{ }^{* *} p<0.01$ (Student's $t$-test)

STING is required for the $\mathrm{CD}^{+} \mathrm{T}$ cell proliferation activated by irradiated EG7 cells and is important in the irradiated tumor cell vaccine eliciting effective antitumor immunity.

However, TLR9 (Toll-like receptor 9) and NLRP3 (Nod-like receptor-family pyrin domain containing 3 ) may also play roles in innate immune signaling by recognizing intracellular DNA, such as mitochondrial DNA. ${ }^{46}$ To investigate whether TLR9 is required to mediate the antitumor response induced by the irradiated cell vaccine, EG7 tumor cells were implanted in the flanks of WT and $\mathrm{Tl} r 9^{-1-}$ mice after three immunizations with the irradiated EG7 cell vaccine. Tumor growth inhibition was comparable between the WT and $\mathrm{Tl}^{-1-} \mathrm{I}^{-1}$ mice (Fig. 7f), demonstrating that host TLR9 may not be required for the antitumor effect induced by the irradiated vaccine. In addition, IL-1 $\beta$ expression induced by AIM2 (absent in melanoma 2), TLR9 and NLRP3 plays an important role in the immune response to DNA. ${ }^{46,47}$ To examine whether IL-1 $\beta$ is involved in the antitumor effect of the vaccine, EG7 cells were implanted in WT and $I L-1 \beta^{-/-}$mice after immunizations. The absence of host IL-1 $\beta$ also did not affect the antitumor activity induced by the irradiated vaccine (Fig. $7 g$ ), suggesting that IL-1 $\beta$ secretion may not be required. Taken together, these results demonstrate that oxidized mtDNA-STING signaling but not TLR9 signaling or IL-1 $\beta$ secretion is involved in the protective antitumor immunity induced by the irradiated cancer cell vaccine.

\section{DISCUSSION}

lonizing radiation, a physical treatment to inactivate live tumor cells, has been extensively applied to enhance the antitumor effects of cancer cell vaccines in both animal research and human clinical trials. ${ }^{4-8}$ Nevertheless, the mechanisms by which irradiated cells function as an immunogenic tumor vaccine and induce an effective antitumor response have not been fully explored. In the present study, we found that the mtDNA-STING pathway was important for the protective antitumor effect of an irradiated cancer cell vaccine, while TLR9 and IL-1 $\beta$ signaling was not. X-ray irradiation induced tumor cell apoptosis and functioned as an effective vaccination strategy via lethal stimulation. In addition, ROS induced by X-ray irradiation produced $8-\mathrm{OHG}$, resulting in oxidative mtDNA damage in EG7 cells. The oxidation of 8-OHG protects oxidized DNA strands from TREX1 degradation. ${ }^{24}$ Moreover, DCs engulfed greater numbers of irradiated EG7 cells than untreated EG7 cells. Oxidized mtDNA derived from irradiated tumor cells gained access to the cytosol of DCs, which activated STING-TBK1-IRF3 signaling in the DC cytoplasm. STING is a critical mediator of IFN- $\beta$ induction mediated by intracellular exogenous DNA in DCs, a type of antigen-presenting cell (APC), thus enhancing the cross-presentation of apoptotic EG7 cell-derived antigens after irradiation. Moreover, loaded DCs cocultured with apoEG7 cells potentiated the proliferation of $\mathrm{CD}^{+} \mathrm{T}$ cells after 


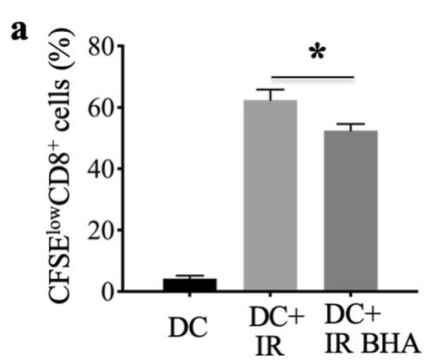

b

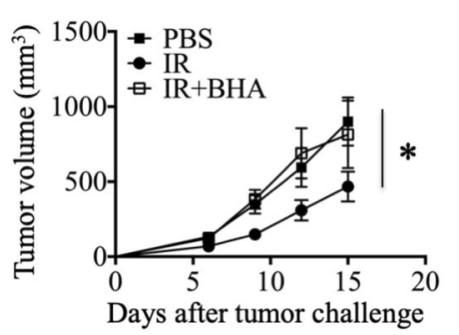

c

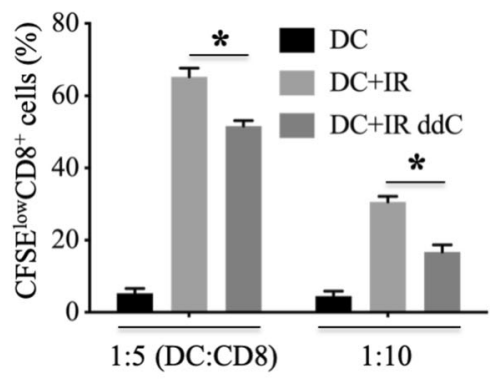

d

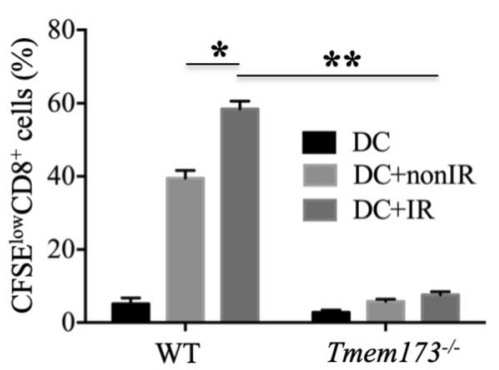

f

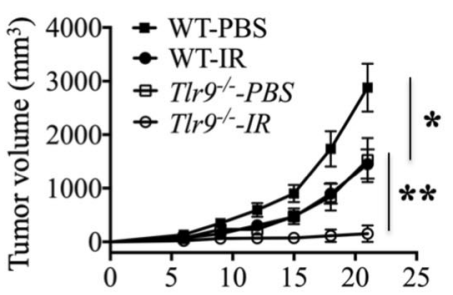

Days after tumor challenge e

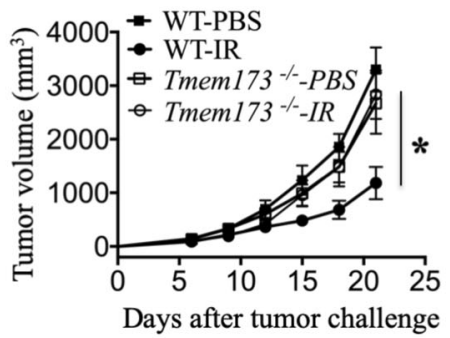

g

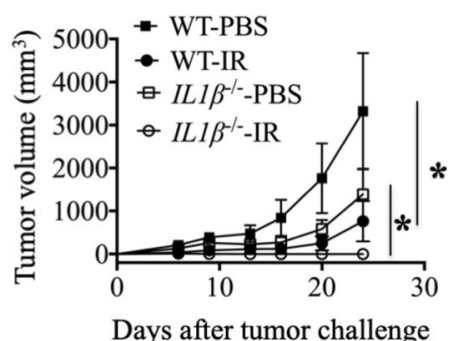

Fig. 7 The oxidized mtDNA-STING pathway is required for the antitumor effect of the irradiated tumor cell vaccine. a, $c$ As described in Fig. 6e, EG7 cells treated with BHA-plus irradiation (a) or ddC-plus irradiation (c) were cultured with BMDCs from WT mice (WTDCs) for $18 \mathrm{~h}$. In c the ratio of DCs to $C D 8^{+}$T cells is 1:5 or 1:10. The ratio of DCs to $C D 8^{+}$T cells was 1:5 in $\mathbf{a}$, d, Fig. 6 e. We estimated the proliferation of $C D 8^{+} \mathrm{T}_{\text {cells }}$ by measuring the percentages of $\mathrm{CFSE}^{\text {low }} \mathrm{CD}^{+}$cells in $\mathrm{CD} 8^{+} \mathrm{T}$ cells by flow cytometry. $\mathbf{b}$ The growth of subcutaneous EG7 cells after preventive vaccination with PBS, irradiated EG7 cells or BHA-plus irradiated EG7 cells was monitored. $\mathbf{d}$ The proliferation of CD8 ${ }^{+} \mathrm{T}$ cells cultured with WTDCs or Tmem $173^{-1-}$ DCs is shown. Tmem $173^{-1-}$ represents STING-deficient mice. e Tumor growth was monitored in WT and Tmem $173^{-1-}$ mice after preventive vaccination with PBS or irradiated EG7 cells. f Tumor growth was monitored in WT and Tlr9 ${ }^{-/-}$mice after vaccination. g Tumor growth was monitored in WT and $I L-1 \beta^{-1-}$ mice after vaccination. Representative data are shown for three (a-g) experiments conducted with 6-8 (b, e-g) mice per group. Data are represented as the mean \pm SEM. ${ }^{*} p<0.05,{ }^{* *} p<0.01$ (Student's $t$-test)

stimulation in a STING pathway-dependent manner. We also observed that the ROS scavenger BHA inhibited the antitumor effect of the irradiated tumor cell vaccine by impairing oxidative mtDNA damage and $\mathrm{CD}^{+} \mathrm{T}$ cell proliferation. Based on our findings, we demonstrate that the elevations in ROS and mtDNA 8-OHG content can be induced within irradiated tumor cells and oxidized mtDNA is transferred to the cytoplasm of DCs. Furthermore, the STING pathway in DCs, which is activated by oxidized mtDNA, is critical for eliciting the antitumor immunity mediated by the irradiated tumor cell vaccine (Fig. 8).

In the present study, we observed that mtDNA, i.e., a DAMP, played an important role in the protective antitumor response induced by the irradiated immunogenic tumor cell vaccine. However, the application of different X-ray doses, which may potentially result in different amounts of mtDNA leakage from the irradiated cells, did not correlate with the antitumor response induced by the irradiated cell vaccine. It is possible that the intact cell membrane retained during apoptosis, which is the major type of cell death after irradiation, results in minimal mtDNA leakage and limited stimulation of host immune cells. In addition, X-rays induce tumor cells to generate elevated amounts of ROS, which results in subsequent oxidative DNA damage (i.e., 8-OHG), and these data are consistent with previous data obtained with UV irradiation. ${ }^{24}$ Given that mtDNA lacks the repair proteins and protective histones found in nDNA, mtDNA is more easily attacked by $\mathrm{ROS}^{22}$ which was confirmed after exposure of cells to $5 \mathrm{~Gy}$ gamma-ray irradiation. ${ }^{21}$ In our study, we found that X-rayirradiated EG7 tumor cells exhibited intense labeling in the peripheral nuclear region with minimal $8-\mathrm{OHG}$ content in the central area in vitro and in vivo and that oxidized 8-OHG colocalized with MitoTracker fluorescence, which labeled mitochondria. In addition, the 8-OHdG content in mtDNA derived from apoEG7 cells was markedly increased. Cell-surface exposure of CRT is critical for establishing the immunogenicity of tumor cell death elicited by irradiation, which is involved in the enhanced phagocytosis of irradiated cells by DCs. ${ }^{7,16}$ Our results revealed that DCs could engulf more irradiated EG7 cells than untreated EG7 cells and that oxidized mtDNA was delivered to the cytoplasm of DCs after irradiated tumor cell vaccination and subsequently elicited antitumor immunity. Our study does not exclude the possibility that other DAMPs, such as other mitochondrial components (proteins, peptides, cytochrome c, ATP, etc.), or nDNA can efficiently activate the STING pathway, but it demonstrates that mtDNA is a major initiator of the maximal antitumor effect induced by the irradiated cell vaccine. Moreover, DNA vaccines have emerged as an attractive immunotherapeutic strategy against cancer due to their simplicity, stability, and safety. ${ }^{48}$ It is possible to design oxidized mtDNA for use as an adjuvant to enhance the antitumor efficacy of cancer vaccines, including DNA vaccines, cell vaccines, and DC vaccines. 


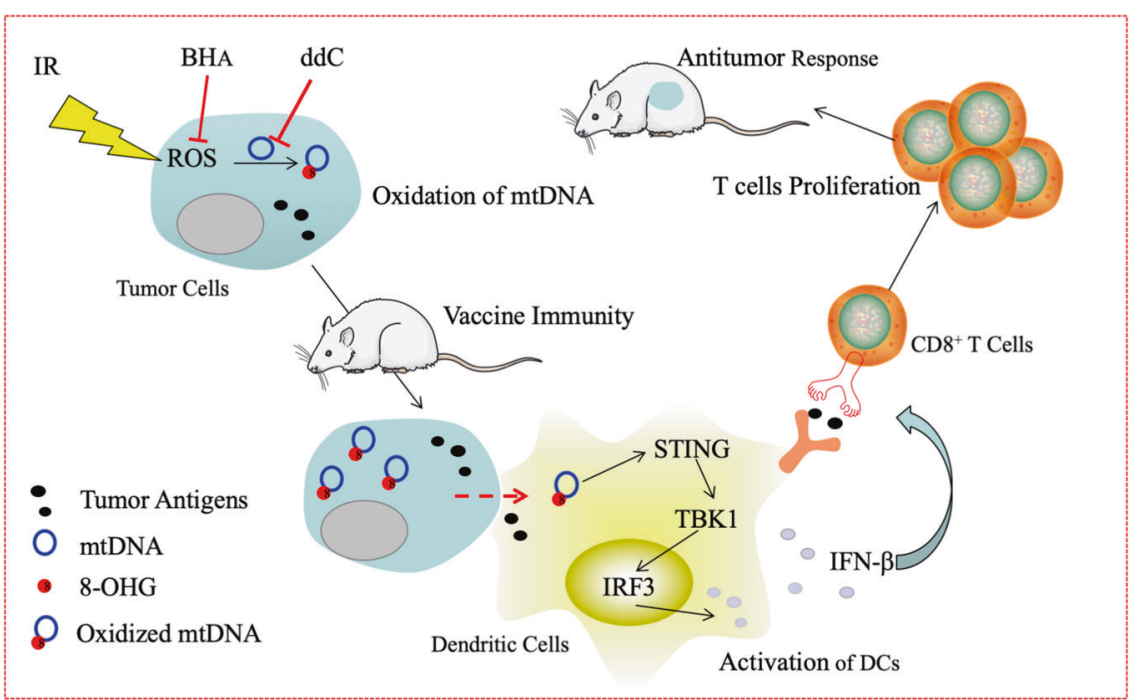

Fig. 8 Oxidized mitochondrial DNA from irradiated tumor cells gains access to the cytoplasm of dendritic cells, subsequently activating the STING-TBK1-IRF3-type I interferon pathway and eliciting antitumor immunity. Irradiation induces tumor cell apoptosis, ROS release, and upregulation of the 8-OHG content, which results in oxidative mtDNA damage in tumor cells. After immunization with an irradiated tumor cell vaccine, oxidized mtDNA derived from tumor cells is transferred to the cytoplasm of DCs, which in turn activates STING-TBK1-IRF3 signaling in the DC cytoplasm and induces type I interferon production. Type I interferons enhance the cross-presentation of apoptotic tumor cell-derived antigens after irradiation and potentiate the proliferation of $\mathrm{CD} 8^{+} \mathrm{T}$ cells, leading to protective antitumor immunity. Moreover, a ROS scavenger (BHA) and an inhibitor of mtDNA polymerase $\gamma(\mathrm{ddC})$ have the ability to impair $\mathrm{CD}^{+} \mathrm{T}$ cell proliferation by inhibiting oxidative mtDNA damage

Our study found that the mtDNA-STING pathway is critical for potentiating the effective antitumor immunity triggered by an irradiated cell vaccine. The mechanisms by which ionizing radiation can potentiate the antitumor response induced by either an irradiated tumor cell vaccine or local radiotherapy have been explored but remain largely unknown. ${ }^{12,14-17}$ It has been reported that the cytosolic DNA-STING pathway and canonical NF-KB pathway play important roles in the response to local irradiation. ${ }^{15,18}$ However, these studies did not address the potential role of mitochondrial DNA or oxidized mitochondrial DNA in the activation of the STING pathway in response to locally irradiated tumor cells. Our research is focused on an irradiated cell vaccine, not local irradiation. However, the mechanism underlying the response to local irradiation mentioned above may be partially involved in the response elicited by our irradiated cell vaccine. The host protein STING has been identified as a central signaling molecule in the innate immune response to cytosolic DNA. ${ }^{45}$ In addition, enhanced immune sensing, which is induced by oxidized genomic DNA damaged by UV irradiation, is dependent on STING signaling. ${ }^{24}$ More recently, oxidized mtDNA was reported to participate in the STING-type I IFN axis in lupus. ${ }^{26,27}$ In our study, we highlight the role of the oxidized mtDNA-STING pathway in eliciting antitumor immunity in response to our irradiated tumor cell vaccine. Moreover, a recent study ${ }^{49}$ indicated that irradiated GM-CSF-producing cellular cancer vaccines formulated with a STING agonist cure established tumors resistant to PD-1 (programmed death ligand 1) blockade. STING signaling may represent a more attractive approach for immunotherapy or for synergy with radiotherapy ${ }^{50}$ or targeted therapy in clinical cancer patients.

Our study reveals a mechanism wherein oxidized mtDNA derived from irradiated tumor cells is delivered to the cytosol of DCs. In addition, oxidation of 8-OHG induced by $\mathrm{X}$-ray irradiation maintains the stability of mtDNA in the cytosol of DCs. However, additional details regarding how irradiated tumor DNA is released into the cytosol of DCs and interacts with DNA sensors must be investigated further. Our study does not exclude the possibility that oxidized tumor-derived mtDNA gains access to the cytoplasm of DCs via phagocytosis when DCs engulf irradiated tumor cells. Another possible mechanism involves the CLEC9A receptor, which can mediate uptake of material from dying cells by $\mathrm{CD}^{+} \mathrm{a}^{+} \mathrm{DCs}$ and promote crosspresentation of dead cell-associated antigens to $C D 8^{+} \mathrm{T}$ cells. ${ }^{51} \mathrm{~A}$ third possibility is that tumor cell-derived DNA is transferred directly from the cytoplasm of irradiated tumor cells to the cytoplasm of DCs through gap junctions composed of connexin 43 (CX43) in a contactdependent manner, a mechanism that is known to participate in antigen cross-presentation. ${ }^{52}$ Another candidate mechanism of DNA transfer involves binding to the antimicrobial peptide LL37, which leads to DNA escape from autophagy and degradation by DNase II. ${ }^{53,54}$ Several studies have reported that the DNA-LL37 complex activates the STING pathway. ${ }^{24,53}$

In summary, our findings reveal a molecular mechanism wherein oxidized mtDNA derived from irradiated tumor cells gains access to the cytosol of DCs. Oxidized mtDNA, as a DAMP or adjuvant, activates the STING-TBK1-IRF3-IFN- $\beta$ pathway in DCs, which is followed by cross-presentation of irradiated tumor cellderived antigens to $\mathrm{CD} 8^{+} \mathrm{T}$ cells and potentiation of the antitumor effect of the irradiated immunogenic tumor cell vaccine.

\section{ACKNOWLEDGEMENTS}

This work was supported by the National Natural Science Foundation Regional Innovation and Development (No. U19A2003), National Major Scientific and Technological Special Project for "Significant New Drugs Development" (No. 2018ZX09733001), Excellent Youth Foundation of the Sichuan Scientific Committee Grant in China (No. 2019JDJQ008) and Development Program of China (No. 2016YFA0201402)

\section{AUTHOR CONTRIBUTIONS}

X.W.W. and C.J.F. conceived and designed the study. C.J.F., J.D., L.L., F.M., and F.F.N. performed the experiments. M.L., W.W., K.M., and H.S.Y. contributed resources. C.J.F., J.D., and L.L. analyzed the data. C.J.F. and X.W.W. wrote the manuscript.

\section{ADDITIONAL INFORMATION}

The online version of this article (https://doi.org/10.1038/s41423-020-0456-1) contains supplementary material.

Competing interests: The authors declare no competing interests. 


\section{REFERENCES}

1. Kantoff, P. W. et al. Sipuleucel-T immunotherapy for castration-resistant prostate cancer. N. Engl. J. Med. 363, 411-422 (2010).

2. Schwartzentruber, D. J. et al. gp100 peptide vaccine and interleukin-2 in patients with advanced melanoma. N. Engl. J. Med. 364, 2119-2127 (2011).

3. Keenan, B. P. \& Jaffee, E. M. Whole cell vaccines-past progress and future strategies. Semin. Oncol. 39, 276-286 (2012).

4. Duraiswamy, J., Kaluza, K. M., Freeman, G. J. \& Coukos, G. Dual blockade of PD-1 and CTLA-4 combined with tumor vaccine effectively restores T-cell rejection function in tumors. Cancer Res. 73, 3591-3603 (2013).

5. Hunn, M. K. et al. Vaccination with irradiated tumor cells pulsed with an adjuvant that stimulates NKT cells is an effective treatment for glioma. Clin. Cancer Res. 18, 6446-6459 (2012).

6. Mortara, L., Frangione, V., Castellani, P., De Lerma Barbaro, A. \& Accolla, R. S. Irradiated CIITA-positive mammary adenocarcinoma cells act as a potent antitumor-preventive vaccine by inducing tumor-specific $\mathrm{CD}^{+}{ }^{+} \mathrm{T}$ cell priming and $\mathrm{CD}^{+}{ }^{+} \mathrm{T}$ cell effector functions. Int. Immunol. 21, 655-665 (2009).

7. Obeid, M. et al. Calreticulin exposure is required for the immunogenicity of gamma-irradiation and UVC light-induced apoptosis. Cell Death Differ. 14, 1848-1850 (2007).

8. Vermorken, J. B. et al. Active specific immunotherapy for stage II and stage III human colon cancer: a randomised trial. Lancet 353, 345-350 (1999).

9. Bonner, J. A. et al. Radiotherapy plus cetuximab for squamous-cell carcinoma of the head and neck. N. Engl. J. Med. 354, 567-578 (2006).

10. Begg, A. C., Stewart, F. A. \& Vens, C. Strategies to improve radiotherapy with targeted drugs. Nat. Rev. Cancer 11, 239-253 (2011).

11. Liauw, S. L., Connell, P. P. \& Weichselbaum, R. R. New paradigms and future challenges in radiation oncology: an update of biological targets and technology. Sci. Transl. Med. 5, 173sr172 (2013).

12. Postow, M. A. et al. Immunologic correlates of the abscopal effect in a patient with melanoma. N. Engl. J. Med. 366, 925-931 (2012).

13. Demaria, $\mathrm{S}$. et al. Ionizing radiation inhibition of distant untreated tumors (abscopal effect) is immune mediated. Int. J. Radiat. Oncol. 58, 862-870 (2004).

14. Reits, E. A. et al. Radiation modulates the peptide repertoire, enhances MHC class I expression, and induces successful antitumor immunotherapy. J. Exp. Med. 203, 1259-1271 (2006).

15. Deng, L. et al. STING-dependent cytosolic DNA sensing promotes radiationinduced type I interferon-dependent antitumor immunity in immunogenic tumors. Immunity 41, 843-852 (2014)

16. Perez, C. A., Fu, A., Onishko, H., Hallahan, D. E. \& Geng, L. Radiation induces an antitumour immune response to mouse melanoma. Int. J. Radiat. Biol. 85, 1126-1136 (2009).

17. Chakraborty, M. et al. Irradiation of tumor cells up-regulates Fas and enhances CTL lytic activity and CTL adoptive immunotherapy. J. Immunol. 170, 6338-6347 (2003).

18. Hou, Y. et al. Non-canonical NF-kappaB antagonizes STING sensor-mediated DNA sensing in radiotherapy. Immunity 49, 490-503 (2018).

19. Seong, S. Y. \& Matzinger, P. Hydrophobicity: an ancient damage-associated molecular pattern that initiates innate immune responses. Nat. Rev. Immunol. 4, 469-478 (2004)

20. Yu, E. P., Bennett, M. R. Mitochondrial DNA damage and atherosclerosis. Trends Endocrinol. Metab. 25, 481-487 (2014).

21. Yoshida, T., Goto, S., Kawakatsu, M., Urata, Y. \& Li, T. S. Mitochondrial dysfunction, a probable cause of persistent oxidative stress after exposure to ionizing radiation. Free Radic. Res. 46, 147-153 (2012).

22. Sabharwal, S. S., Schumacker, P. T. \& Mitochondrial, R. O. S. in cancer: initiators, amplifiers or an Achilles' heel? Nat. Rev. Cancer 14, 709-721 (2014).

23. Moeller, B. J., Cao, Y., Li, C. Y. \& Dewhirst, M. W. Radiation activates HIF-1 to regulate vascular radiosensitivity in tumors: role of reoxygenation, free radicals, and stress granules. Cancer Cell 5, 429-441 (2004).

24. Gehrke, N. et al. Oxidative damage of DNA confers resistance to cytosolic nuclease TREX1 degradation and potentiates STING-dependent immune sensing. Immunity 39, 482-495 (2013).

25. West, A. P. et al. Mitochondrial DNA stress primes the antiviral innate immune response. Nature 520, 553-557 (2015)

26. Caielli, S. et al. Oxidized mitochondrial nucleoids released by neutrophils drive type I interferon production in human lupus. J. Exp. Med. 213, 697-713 (2016).

27. Lood, C. et al. Neutrophil extracellular traps enriched in oxidized mitochondrial DNA are interferogenic and contribute to lupus-like disease. Nat. Med. 22, 146-153 (2016)

28. Illner, D. \& Scherthan, H. lonizing irradiation-induced radical stress stalls live meiotic chromosome movements by altering the actin cytoskeleton. Proc. Natl Acad. Sci. USA 110, 16027-16032 (2013).

29. Tait, S. W. et al. Widespread mitochondrial depletion via mitophagy does not compromise necroptosis. Cell Rep. 5, 878-885 (2013).
30. Wang, Q. et al. Reactive oxygen species-mediated apoptosis contributes to chemosensitization effect of saikosaponins on cisplatin-induced cytotoxicity in cancer cells. J. Exp. Clin. Cancer Res. 29, 159 (2010).

31. Marichal, T. et al. DNA released from dying host cells mediates aluminum adjuvant activity. Nat. Med. 17, 996-1002 (2011).

32. Wei, X. et al. Cationic nanocarriers induce cell necrosis through impairment of $\mathrm{Na}$ $(+) / \mathrm{K}(+)$-ATPase and cause subsequent inflammatory response. Cell Res. 25, 237-253 (2015).

33. Eaton, J. S., Lin, Z. P., Sartorelli, A. C., Bonawitz, N. D. \& Shadel, G. S. Ataxiatelangiectasia mutated kinase regulates ribonucleotide reductase and mitochondrial homeostasis. J. Clin. Investig. 117, 2723-2734 (2007).

34. Nakahira, K. et al. Autophagy proteins regulate innate immune responses by inhibiting the release of mitochondrial DNA mediated by the NALP3 inflammasome. Nat. Immunol. 12, 222-230 (2011).

35. Zhang, Z. et al. The helicase DDX41 senses intracellular DNA mediated by the adaptor STING in dendritic cells. Nat. Immunol. 12, 959-965 (2011).

36. Lorenzi, S. et al. Type I IFNs control antigen retention and survival of CD8alpha(+) dendritic cells after uptake of tumor apoptotic cells leading to cross-priming. J. Immunol. 186, 5142-5150 (2011).

37. Deng, L. et al. Irradiation and anti-PD-L1 treatment synergistically promote antitumor immunity in mice. J. Clin. Investig. 124, 687-695 (2014).

38. Corrales, L. et al. Direct activation of STING in the tumor microenvironment leads to potent and systemic tumor regression and immunity. Cell Rep. 11, 1018-1030 (2015).

39. Woo, S. R. et al. STING-dependent cytosolic DNA sensing mediates innate immune recognition of immunogenic tumors. Immunity 41, 830-842 (2014).

40. Chen, T. Y., Chi, K. H., Wang, J. S., Chien, C. L. \& Lin, W. W. Reactive oxygen species are involved in FasL-induced caspase-independent cell death and inflammatory responses. Free Radic. Biol. Med. 46, 643-655 (2009).

41. Wiseman, H. \& Halliwell, B. Damage to DNA by reactive oxygen and nitrogen species: role in inflammatory disease and progression to cancer. Biochem. J. 313, 17-29 (1996).

42. Kaguni, L. S. DNA polymerase gamma, the mitochondrial replicase. Annu. Rev. Biochem. 73, 293-320 (2004).

43. Rongvaux, A. et al. Apoptotic caspases prevent the induction of type I interferons by mitochondrial DNA. Cell 159, 1563-1577 (2014).

44. Sun, L., Wu, J., Du, F., Chen, X. \& Chen, Z. J. Cyclic GMP-AMP synthase is a cytosolic DNA sensor that activates the type I interferon pathway. Science 339, 786-791 (2013).

45. Burdette, D. L. \& Vance, R. E. STING and the innate immune response to nucleic acids in the cytosol. Nat. Immunol. 14, 19-26 (2013).

46. Fang, C., Wei, X., Wei, Y. \& Mitochondrial, D. N. A. in the regulation of innate immune responses. Protein Cell https://doi.org/10.1007/s13238-015-0222-9 (2015).

47. Barber, G. N. Cytoplasmic DNA innate immune pathways. Immunol. Rev. 243, 99-108 (2011).

48. Yang, B., Jeang, J., Yang, A., Wu, T. C. \& Hung, C. F. DNA vaccine for cancer immunotherapy. Hum. Vacc. Immunotherap. 10, 3153-3164 (2014).

49. Fu, J. et al. STING agonist formulated cancer vaccines can cure established tumors resistant to PD-1 blockade. Sci. Transl. Med. 7, 283 ra252 (2015).

50. Shevtsov, M., Sato, H., Multhoff, G. \& Shibata, A. Novel approaches to improve the efficacy of immuno-radiotherapy. Front. oncol. 9, 156 (2019).

51. Sancho, D. et al. Identification of a dendritic cell receptor that couples sensing of necrosis to immunity. Nature 458, 899-903 (2009).

52. Neijssen, J. et al. Cross-presentation by intercellular peptide transfer through gap junctions. Nature 434, 83-88 (2015).

53. Chamilos, G. et al. Cytosolic sensing of extracellular self-DNA transported into monocytes by the antimicrobial peptide LL37. Blood 120, 3699-3707 (2012).

54. Zhang, Z. et al. Mitochondrial DNA-LL-37 complex promotes atherosclerosis by escaping from autophagic recognition. Immunity 43, 1137-1147 (2015).

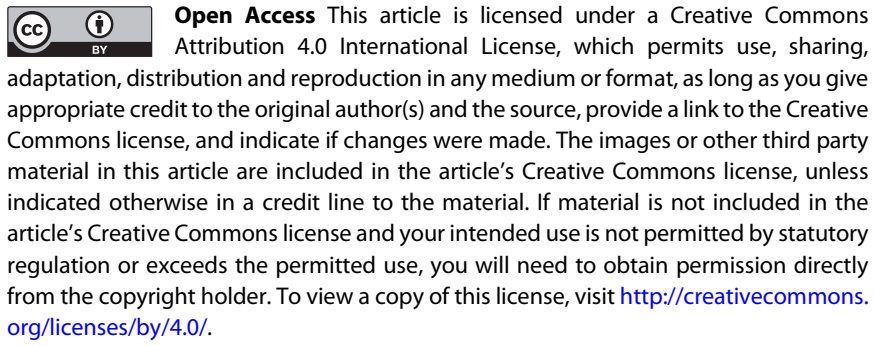

(c) The Author(s) 2020 